\title{
Statistical Quality Control with the qcr Package
}

\author{
by Miguel Flores, Rubén Fernández-Casal, Salvador Naya, Javier Tarrío-Saavedra
}

\begin{abstract}
The R package qcr for Statistical Quality Control (SQC) is introduced and described. It includes a comprehensive set of univariate and multivariate SQC tools that completes and increases the SQC techniques available in R. Apart from integrating different R packages devoted to SQC (qcc, MSQC), qcr provides nonparametric tools that are highly useful when Gaussian assumption is not met. This package computes standard univariate control charts for individual measurements, $\bar{x}, S, R$, $p, n p, c, u$, EWMA, and CUSUM. In addition, it includes functions to perform multivariate control charts such as Hotelling $\mathrm{T}^{2}$, MEWMA and MCUSUM. As representative features, multivariate nonparametric alternatives based on data depth are implemented in this package: $r, Q$ and $S$ control charts. The qcr library also estimates the most complete set of capability indices from first to the fourth generation, covering the nonparametric alternatives, and performing the corresponding capability analysis graphical outputs, including the process capability plots. Moreover, Phase I and II control charts for functional data are included.
\end{abstract}

\section{Introduction}

Throughout the last decades, there has been an increasing interest to measure, improve, and control the quality of products, services, and procedures. This is connected to the strong relationship between quality, productivity, prestige, trust, and brand image. In fact, implementing procedures of statistical quality control (SQC) is currently related to increasing companies' competitiveness.

The concept of quality control has been extended from the first definitions based on the idea of adjusting production to a standard model to satisfy customer requirements and include all participants. Nowadays, SQC is not only applied to manufactured products but to all industrial and service processes.

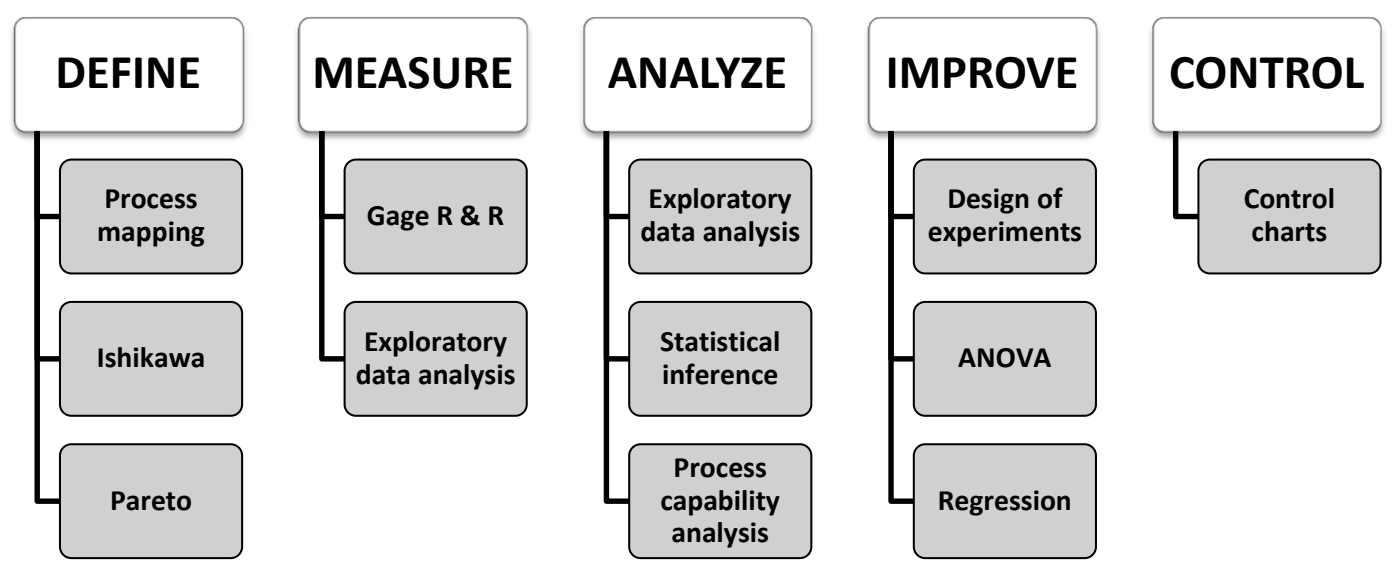

Figure 1: Statistical tools applied in each steps of the Six Sigma methodology.

The use of different SQC techniques was standardized With the development of the Six Sigma method by Motorola in 1997 (Pande et al., 2000). Six Sigma is a methodology or even philosophy focused on variability reduction that promotes the use of statistical methods and tools in order to improve processes in industry and services. The Six Sigma application is composed of five stages: Define, Measure, Analyze, Improve, and Control (DEMAIC). Figure 1 shows some representative statistical techniques applied in each of the Six Sigma stages. The two most representative statistical tools of SQC are the control charts and the process capability analysis (Montgomery, 2009). Therefore, the proposed qcr package has been developed in order to provide users a comprehensive and free set of programming tools to apply control charts and perform capability analysis in the SQC framework.

The control stage is characterized by the use of tools based on anomaly detection and correction (Montgomery, 2009). The most representative techniques of this stage and the primary tool of the Statistical Process Control (SPC) are the control charts (Champ and Woodall, 1987). They have been 
developed to evaluate the process performance and at any time. The use of control charts prevents the process from getting out of control, and helping to detect the assignable causes corresponding to variations of the critical-to-quality features (CTQs), thus performing process changes when actually required. Furthermore, control charts provide estimates of the natural range of process variation (natural control limits), allowing us to compare this range with those limits specified by standards, company managers, or customers (specification limits). Hence, the process monitoring can be carried out by comparing each new observation with these natural limits, preventing defects in the final product. Briefly, a control chart is a two-dimensional graph whose axis represents the variable or attribute that is being monitored (CTQ variables). The estimation of natural control limits of the CTQ variables is developed by a process composed of two phases: In Phase I, the natural control limits are estimated using a preliminary sample (calibration sample) where we assume that the causes of variation are only random. In Phase II, each new observation is plotted on the control chart along with the natural limits obtained in the previous step. The set of new observations (twhich are not used to calculate the natural control limits) make up the so-called monitoring sample. Patterns, observations of out of control limits, runs of more than six observations on one side of the central line, among others, are some of the different criteria to identify out of control states in a specific process, providing also valuable information about the detection of any assignable causes of variation in the monitoring.

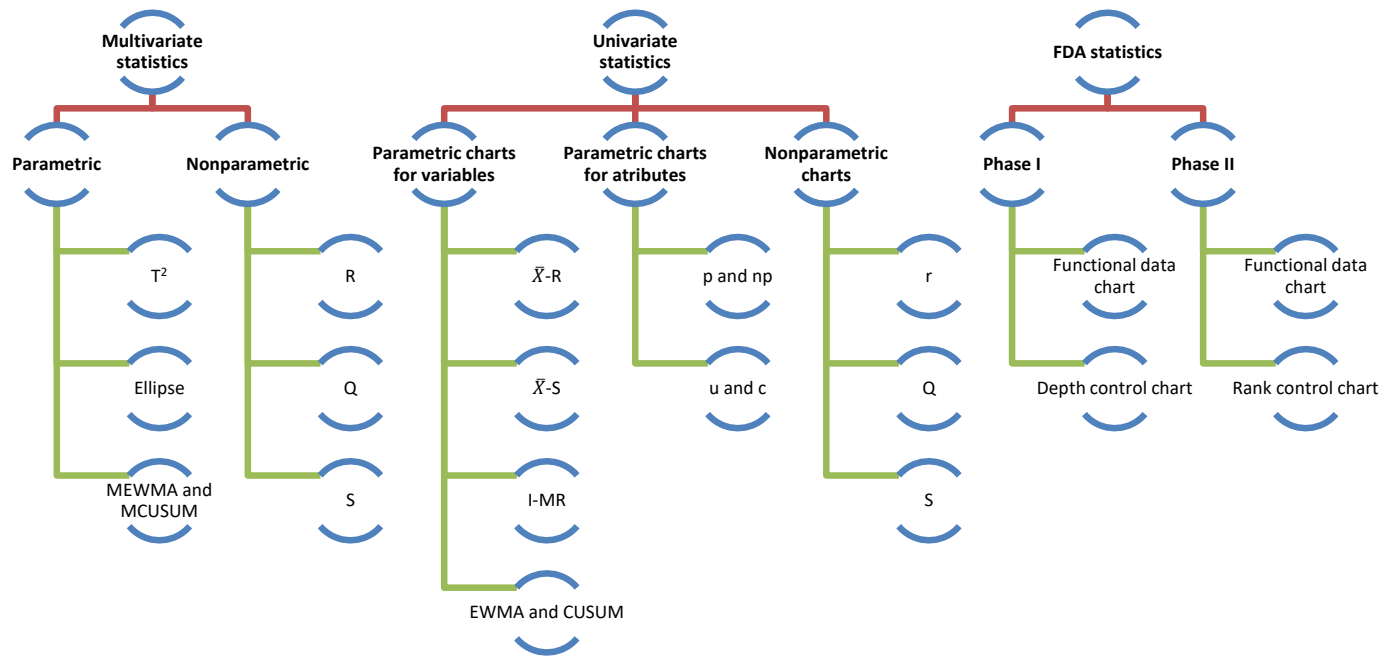

Figure 2: Control charts implemented in the qcr package.

The most used control charts are based on the assumptions of normality and independence of the studied CTQ variables. These charts are used to control the position and dispersion of CTQ attributes and variables. Figure 2 shows some of the most important types of control charts. These can be classified according to the type of feature that is being controlled (attribute or variable), the variable dimension (univariate or multivariate), and assuming or not a parametric distribution of the variable (parametric or nonparametric). The qcr package provides charts for the mean $(\bar{x})$, standard deviation $(s)$, range $(R)$, individual measurements $(I)$, moving ranges $(M R)$, proportion of nonconforming units $(p)$, number of nonconforming units $(n p)$, number of defects per unit $(c)$, mean number of defects per control unit $(u)$, exponentially weighted moving average (EWMA), and cumulative sum control chart (CUSUM). The last two techniques are also called memory control charts, and they are specially designed to detect shifts of less than two standard deviations, both when using rational samples or individual measurements. On the other hand, new control charts based on the concept of data depth and developed by Liu (1995) are implemented in qcr. Those are the $r, Q$, and $S$ control charts, the nonparametric alternatives for individual measurements, mean control chart, and CUSUM control chart, respectively. When more than one variable defines the process quality, multivariate control charts are applied. If the Gaussian assumption is met, the Hotelling $\mathrm{T}^{2}$ control chart can be applied. If we want to detect small deviations, multivariate EWMA (MEWMA) and multivariate CUSUM (MCUSUM) can be implemented. When no parametric distribution is assumed, $r, Q$, and $S$ charts can be used.

Another interesting SQC tool, which is very useful in the industry, is the Process Capability Analysis (PCA). It estimates how well a process meets the tolerances defined by the company, customers, standards, etc., by comparing the specification tolerances with respect to the natural range of variation of CTQ features. The capability of the process is measured using capability 
indicators. Process Capability Ratio (PCR) is a numerical score that helps the manufacturers know whether the output of a process meets the engineering specifications. Large PCR values show that the industrial or service process is capable of meeting the customer requirements. There have been many different PCRs developed in the last four decades that require the Gaussian assumption for the CTQ variable (Boyles, 1991). However, many processes in industry and real applications do not meet this hypothesis. Thus, we could innacuratelly estimate the capability using PCR. Hence, many authors have studied different nonparametric alternatives to traditional PCR (Polansky, 2007).

The qcr package has been developed in R (R Core Team, 2021) under the GNU license. Nowadays, there are other $\mathrm{R}$ packages that currently provide quality control tools for users. The use of each one is shown in Figure 3.

The qcc package (Scrucca, 2004) was developed by Professor Luca Scrucca of the Department of Economics, Finance, and Statistics at the University of Perugia. It enables us to perform Shewhart quality control charts for variables and attributes, as well as the CUSUM and EWMA charts for detecting small changes in the CTQ variable. Multivariate analysis is performed applying the Hotelling $\mathrm{T}^{2}$ control chart. Additionally, it has functions implemented to obtain the operating characteristic curves $(\mathrm{OC})$ and to estimate process capability analysis indices. Pareto and Ishikawa diagrams are also implemented. Otherwise, the IQCC package (Barros, 2017) is maintained by Professor Emanuel P. Barbosa of the Institute of Mathematics in the State University of Campinas. It has a smaller number of control charts implemented, but it incorporates multivariate graphics. The qualityTools package (Roth, 2016) was developed to aid learning in quality sciences. Figure 3 shows some of its utilities, e.g., capability analysis (providing a comprehensive set of parametric distributions) and design of experiments. In addition, the SixSigma library (Cano et al., 2012, 2015) provides alternative functions to qualityTools and qcc packages and the possibility of implementing process maps.
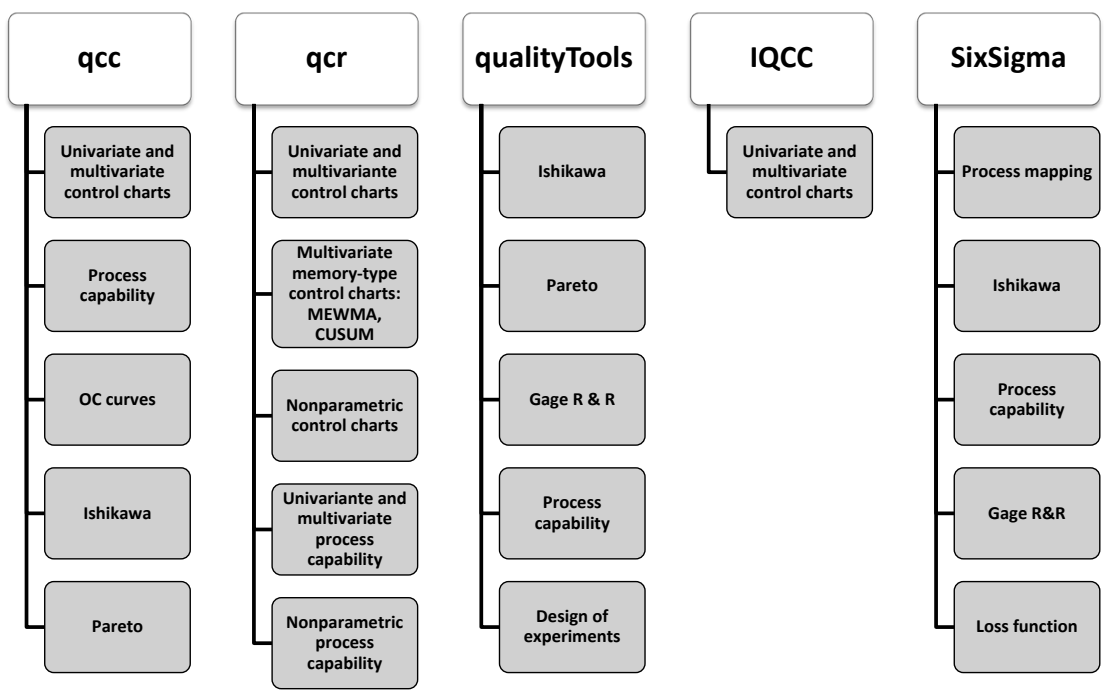

Figure 3: Comparison between the main packages in R devoted to Statistical Quality Control and the qcr package.

Furthermore, there are other libraries specifically focused on control chart applications. Namely, the spcadjust (Gandy and Kvaloy, 2013) that allows us to estimate the calibration control limits of Shewhart, CUSUM, and EWMA control charts, and the spc (Knoth, 2021) which provides tools for the evaluation of EWMA, CUSUM, and Shiryaev-Roberts control charts by using Average Run Length and RL quantiles criteria. Moreover, the MSQC package (Santos-Fernandez, 2013) is a set of tools for multivariate process control, mainly control charts. It contains the main alternatives for multivariate control charts such as Hotelling $\left(\mathrm{T}^{2}\right)$, Chi-squared, MEWMA, MCUSUM, and Generalized Variance control charts. It also includes some tools to evaluate the multivariate normal assumption. The corresponding multivariate capability analysis can be performed using the MPCI library (Santos-Fernández and Scagliarini, 2012) that provides different multivariate capability indices. It is also interesting to mention the edcc package (Zhu and Park, 2013) for its economic design of control charts by minimizing the expected cost per hour of the studied process.

It is important to emphasize that the qcr package also includes new applications such as nonparametric approaches of control charts and capability indices (also covering the capability plots), 
which are currently unavailable in the $\mathrm{R}$ software.

\section{Datasets in the qcr package}

The qcr package contains new databases (see table 1) based on study cases tackled by the authors during their professional activity as well as well-known datasets implemented on other packages focused on statistical quality control such as:

- archery1: It consists of a stage in which the archer shoots 72 arrows. The information is given in $\mathrm{x}$ and $\mathrm{y}$ coordinates. It is implemented in the MSQC package (Santos-Fernandez 2013).

- circuit: Number of nonconformities observed in 26 successive samples of 100 printed circuit boards. It is implemented in the qcc package (Scrucca 2004).

- dowel1: Diameter and length of a dowel pin. It is implemented in the MSQC package (Santos-Fernandez 2013).

- orangejuice: Frozen concentrated orange juice is packed in 6-oz cartons. These cartons are formed on a machine by spinning them from a cardboard stock and attaching a metal bottom panel. A can is then inspected to determine whether, when filled, the liquid could possibly leak either on the side seam or around the bottom joint. If this occurs, a can is considered nonconforming. The data were collected as 30 samples of 50 cans each at half-hour intervals over a three-shift period in which the machine was in continuous operation. It is implemented in the qcc package (Scrucca 2004).

- pcmanufact: A personal computer manufacturer counts the number of nonconformities per unit on the final assembly line. He collects data on 20 samples of 5 computers each. It is implemented in the qcc package (Scrucca 2004).

- pistonrings: Piston rings for an automotive engine are produced by a forging process. The inside diameter of the rings manufactured by the process is measured on 25 samples, each of size 5, drawn from a process being considered 'in control'. It is implemented in the qcc package (Scrucca 2004).

\section{Univariate and multivariate parametric control charts in qcr}

The construction of a control chart is equivalent to the plotting of the acceptance regions of a sequence of hypothesis tests over time. Namely, the $\bar{x}$ chart is a control chart used to monitor the process mean $\mu$. It plots the sample means, $\bar{X}$ 's, corresponding to subgroups of the $\left\{X_{1}, X_{2}, \ldots\right\}$ observations and is equivalent to test the hypotheses $H_{0}: \mu=\mu_{0}$ versus $H_{\alpha}: \mu \neq \mu_{0}$ (for some target value $\left.\mu_{0}\right)$ conducted over time, using $\bar{x}$ as the test statistic. Here we assume that $\left\{X_{1}, X_{2}, \ldots\right\}$ are the sample measurements of a particular CTQ feature that follows the $F$ distribution with mean $\mu$ and standard deviation $\sigma$. When there is insufficient evidence to reject $H_{0}$, we can state that the process is under control; otherwise, the process is out of control. In other words, processes are under control when their sources of variation are only the sources common to the process (Brown and Wetherill, 1990). The decision to reject or not $H_{0}$ is based on the value of the sample mean $\bar{x}$ observed at each time interval (Liu and Tang, 1996). The control charts are easy to construct, visualize, and interpret, and most important, have proven their effectiveness in practice since the 1920's.

Control charts are defined, on the one hand, by a center line that represents the average value of the CTQ feature corresponding to the in-control state and, on the other hand, two horizontal lines, called the upper control limit (UCL) and the lower control limit (LCL). The region between the control limits corresponds to the region where $H_{0}$ is not rejected (defined in the previous section). As a consequence, the process will be out of control when an observed rational sample or an individual measurement falls outside the limits. Let $w$ be a sample statistic that measures a quality characteristic of interest, and suppose that the mean of $w$ is $\mu_{w}$ and the standard deviation of $w$ is $\sigma_{w}$. Then the center line, the upper control limit, and the lower control limit become:

$$
\begin{gathered}
\mathrm{UCL}=\mu_{w}+L \sigma_{w} \\
\mathrm{CL}=\mu_{w} \\
\mathrm{LCL}=\mu_{w}-L \sigma_{w},
\end{gathered}
$$




\begin{tabular}{|c|c|}
\hline Name & Description \\
\hline counters & $\begin{array}{l}\text { A water supply company wants to control the performance of the water } \\
\text { counters installed throughout a city. For this purpose, } 60 \text { rational } \\
\text { samples have been taken, each one composed by } 3 \text { measurements, from } \\
\text { the same age ( } 10 \text { years) and caliber water counters corresponding to } \\
\text { two different brands, and during a period of } 5 \text { years. This dataset } \\
\text { is based on a study case of A Coruña's water supply company, } \\
\text { Empresa Municipal de Aguas de La Coruña (Emalcsa). }\end{array}$ \\
\hline employment & $\begin{array}{l}\text { A Spaniard-Argentinian hotel company wants to control the level of } \\
\text { occupancy (measured in \%) in their establishments through the } \\
\text { application of a continuous control. For this purpose, } 48 \text { subsamples have } \\
\text { been taken from six hotels corresponding to two different countries. }\end{array}$ \\
\hline oxidation & $\begin{array}{l}\text { This database contains information about the resistance against the } \\
\text { oxidation of olive oil of the Picual variety. Five measurements of the } \\
\text { Onset Oxidation Temperature (OOT, index that measures the } \\
\text { resistance against the oxidation) are obtained from } 50 \text { batches of Picual } \\
\text { olive oil produced in chronological order. It is importantly to note } \\
\text { that OOT decreases as the oil is progressively mixed with other } \\
\text { olive oil varieties defined by a lower OOT. }\end{array}$ \\
\hline plates & $\begin{array}{l}\text { A chemical company is developing a patent for a new variant of artificial } \\
\text { stone mostly made of quartz ( } 93 \mathrm{wt} \% \text { and polyester resin). This company } \\
\text { is launching a pilot plant where it begins to produce plates of this } \\
\text { material on an industrial scale. The CTQ variable of this product is the } \\
\text { Vickers hardness. In order to measure the hardness level and hardness } \\
\text { homogeneity of the product, } 50 \text { plates have been measured } 5 \text { times in } \\
\text { different sections. The characteristic learning curves, through gradual } \\
\text { level change, can be observed. }\end{array}$ \\
\hline presion & $\begin{array}{l}\text { A shipyard of recreational boats is intended to optimize and control the } \\
\text { mechanical properties of the yacht hulls made of a composite based on } \\
\text { epoxy resin. In this regard, the modulus of elasticity due to tensile } \\
\text { efforts is measured after applying two different curing pressures: } 0.1 \\
\text { and } 10 \mathrm{MPa} \text {. Overall, } 60 \text { subsamples, composed of three measurements, } \\
\text { obtained from } 60 \text { vessels, have been taken. }\end{array}$ \\
\hline
\end{tabular}

Table 1: Some of the specific datasets included in the qcr package

where $L$ is the "distance" of the control limits from the center line, expressed in standard deviation units.

When several random variables characterize the quality of a process/service, applying statistical multivariate quality control techniques becomes necessary. In fact, if we analyze each variable separately, the probability that an observation of a variable will fall within the calculated limits when it is known that the process is actually under control will no longer be 0.9973 for $6 \sigma$ amplitude. Assuming independence, it will be $0.9973^{p}$, where $p$ is the number of CTQ features, while the probability of type I will actually lead to $\alpha^{\prime}=1-(1-\alpha)^{p}$. Therefore, the control limits are different from those drawn, assuming the control of each CTQ variable independently from the others. Moreover, if the variables are dependent, the calculation of $\alpha$ becomes more complex. This subject is particularly important today, as automatic inspection procedures make it customary to measure many parameters of each product over time. The more common multivariate parametric control charts are the Hotelling $\mathrm{T}^{2}$ (to identify big shifts) and the multivariate CUSUM (MCUSUM) and EWMA (MEWMA) for identifying small shifts.

The functions that compute the quality control statistics for the different univariate control charts (involving continuous, attribute or count data) are shown in Table 2. For the sake of simplicity and taking into account that these types of control charts are implemented in other packages, the use of these functions is not shown in this work. More details are given in the help of qcr package (Flores et al., 2021). 


\begin{tabular}{|c|c|c|}
\hline & & Statistical quality control charts for \\
\hline Function & Chart name & Variables \\
\hline qcs.xbar & $\bar{X}$ & $\begin{array}{l}\text { Sample means of a continuous process variable are } \\
\text { plotted to control the process average. }\end{array}$ \\
\hline qcs. R & $R$ & $\begin{array}{l}\text { Sample ranges of a continuous process variable are } \\
\text { plotted to control the process variability. }\end{array}$ \\
\hline qcs.s & $S$ & $\begin{array}{l}\text { Sample standard deviations of a continuous variable } \\
\text { are plotted to control the process variability. }\end{array}$ \\
\hline qcs.one & $I$ & $\begin{array}{l}\text { Sample values from a I chart data of a continuous } \\
\text { process variable to control the level (position) } \\
\text { of the process. }\end{array}$ \\
\hline & & Attributes \\
\hline$q c s \cdot p$ & $p$ & $\begin{array}{l}\text { Proportion of nonconforming units is plotted, the } \\
\text { number of defective items follow a binomial distribution. }\end{array}$ \\
\hline qcs.np & $n p$ & $\begin{array}{l}\text { Number of nonconforming units is plotted, and the chart } \\
\text { is constructed based on the average of the process. }\end{array}$ \\
\hline qcs.c & $c$ & $\begin{array}{l}\text { Nonconformities per unit are plotted, number of defects } \\
\text { in a large population follow a Poisson distribution. }\end{array}$ \\
\hline qcs.u & $u$ & $\begin{array}{l}\text { Average nonconformities per unit are plotted, this chart } \\
\text { does not require a constant number of units. }\end{array}$ \\
\hline qcs.g & $g$ & $\begin{array}{l}\text { Number of non-events between events are plotted, it } \\
\text { counts the number of events between rarely-occurring } \\
\text { errors or nonconforming incidents. }\end{array}$ \\
\hline & & Attributes and variables \\
\hline qcs.cusum & CUSUM & $\begin{array}{l}\text { Cumulative sums for individual observations or for the } \\
\text { averages of rational subgroups are plotted to monitor } \\
\text { the process mean. }\end{array}$ \\
\hline qcs. ewma & EWMA & $\begin{array}{l}\text { The exponential weighed average of CTQ variables are } \\
\text { plotted to identify small changes in the process } \\
\text { (measured as rational samples or individual observations). } \\
\text { Multivariate control charts }\end{array}$ \\
\hline mqcs.t2 & $\mathrm{T}^{2}$ & $\begin{array}{l}\text { Multivariante Hotelling } \mathrm{T}^{2} \text { control chart for individual } \\
\text { observations (vectors). }\end{array}$ \\
\hline mqcs.mcusum & MCUSUM & $\begin{array}{l}\text { Multivariate Cumulative Sum control chart for individual } \\
\text { observations (vectors). }\end{array}$ \\
\hline mqcs . ewma & MEWMA & $\begin{array}{l}\text { Multivariate EWMA control chart for individual } \\
\text { observations (vectors). }\end{array}$ \\
\hline & & Functional data control charts \\
\hline fdqcs.depth & Phase I & $\begin{array}{l}\text { Phase I control chart for functional data: } \\
\text { depth control chart and deepest curves envelope. }\end{array}$ \\
\hline fdqcs.rank & Phase II & $\begin{array}{l}\text { Phase II control chart for functional data: } \\
\text { rank control chart and deepest curves envelope. }\end{array}$ \\
\hline plot.fdqcs & FDA plots & $\begin{array}{l}\text { Graphical outputs for Phase I and Phase II control charts } \\
\text { for functional data. }\end{array}$ \\
\hline
\end{tabular}

Table 2: Univariate Shewhart, multivariate Hotelling $\mathrm{T}^{2}$, univariate and multivariate CUSUM and EWMA and FDA control charts available in the qcr package

\section{Nonparametric control charts based on data depth}

The control charts presented in this section were proposed by Liu (1995) as an alternative to those described in previous section. The main idea of its control graphs is to reduce each multivariate measure to the univariate index, that is, its relative center-exterior classification induced by a depth of data. This approach is completely nonparametric, and therefore, these control charts are not defined by any parametric assumption regarding the process model. Thus, they are applicable in a wider number of case studies than those counterparts such as $T^{2}$, MCUSUM, and MEWMA control charts. In addition, these graphs allow the simultaneous detection of the change of location (shift of the mean) and the increase of the scale (change in variability) in a process. 
Liu (1995) proposed and justified three types of control charts, the $r, Q$, and $S$ charts which can be considered as data-depth-based multivariate generalizations of the univariate $X, \bar{x}$, and CUSUM charts, respectively.

\section{Data depth}

In multivariate analysis, the term depth refers to the degree of centrality of a point regarding a data cloud or a probability distribution. Therefore, it is possible to define a rank in the multidimensional Euclidean space through the calculation of observation depth. According to Dyckerhoff (2004) and Cascos et al. (2011), the depth function can be defined as a bounded function $D_{p}: R^{d} \longrightarrow R$, with $P$ the distribution set in $R^{d}$, that assigns at each point of $R^{d}$ its degree of centrality with respect to $P$. Depth functions with which control charts can be performed are the

- Simplicial depth (Liu 1990),

- Mahalanobis depth (Mahalanobis 1936),

- Halfspace or Tukey depth (Tukey 1975),

- Likelihood depth (Fraiman et al. 1997), and

- Random projection depth (Zuo and Serfling 2000).

\section{Statistics derived from data depth}

Let $G$ a $k$-dimensional distribution, and let $Y_{1}, \ldots, Y_{m}$ be $m$ random observations from $G$. The sample $Y_{1}, \ldots, Y_{m}$ is generally the reference sample of a CTQ variable in the context of quality control, composed of measurements from products obtained by an under control process. If $X_{1}, X_{2}, \ldots$ are the new observations from the manufacturing process, assuming that the different $X_{i}$ values follow an $F$ distribution if the quality of the studied product has been deteriorated or, in other words, if the process is out of control. Otherwise, they follow a $G$ distribution. Let $D_{G}(\cdot)$ denote a notion of depth, and assume that $G$ and $F$ are two continuous distributions. Thus, if all the $D_{G}\left(Y_{i}\right)$ values are sorted in increasing order, and $Y_{[j]}$ denotes the sample value associated with the $j$ th smallest depth value, then $Y_{[1]}, \ldots, Y_{[m]}$ are the order statistics of $Y_{i}$ 's, with $Y_{[m]}$ being the most central point. Therefore, the smaller the order (or the rank) of a point, the farther that point will be from the underlying distribution $G(\cdot)$.

Liu (1995) defines the rank statistic as

$$
r_{G}(y)=P\left\{D_{G}(Y) \leq D_{G}(y) \mid Y \sim G\right\}
$$

whereby $Y \sim G$ indicates that the random variable $Y$ follows the distribution $G$. When $G$ is unknown, the empirical distribution $G_{m}$ of the sample $\left\{Y_{1}, \ldots, Y_{m}\right\}$ can be used instead, and the statistic is defined by

$$
r_{G_{m}}(y)=\frac{\#\left\{D_{G_{m}}\left(Y_{j}\right) \leq D_{G_{m}}(y), j=1, \ldots, m\right\}}{m}
$$

In the same way that $r_{G}$ and $r_{G_{m}}$, the $Q$ statistics can be also defined as follows

$$
\begin{gathered}
Q(G, F)=P\left\{D_{G}(Y) \leq D_{G}(X) \mid Y \sim G, X \sim F\right\}=E_{F}\left[r_{G}(X)\right] \\
Q\left(G, F_{n}\right)=\frac{1}{n} \sum_{i=1}^{n} r_{G}\left(X_{i}\right) \\
Q\left(G_{m}, F_{n}\right)=\frac{1}{n} \sum_{i=1}^{n} r_{G_{m}}\left(X_{i}\right),
\end{gathered}
$$

whereby $F_{n}(\cdot)$ denotes the empirical distribution of the sample $\left\{X_{1}, \ldots, X_{n}\right\}$. The control charts corresponding to these statistics can be developed as described in the following sections.

\section{The $r$ chart}

Calculate $\left\{r_{G}\left(X_{1}\right), r_{G}\left(X_{2}\right), \ldots, r_{G}\left(X_{n}\right)\right\}$ or $\left\{r_{G_{m}}\left(X_{1}\right), r_{G_{m}}\left(X_{2}\right), \ldots, r_{G_{m}}\left(X_{n}\right)\right\}$ if $G$ is unknown but $Y_{1}, \ldots, Y_{m}$ are available. As a result, the $r$ chart consists of plotting the rank statistic in 
regard to time. The control chart central line is $\mathrm{CL}=0.5$, whereas the lower limit is $\mathrm{LCL}=\alpha$, with $\alpha$ accounting for the false alarm rate. The process will be out of control if $r_{G}(\cdot)$ falls under LCL. A small value of the rank statistic $r_{G m}(X)$ means that only a very small proportion of $Y_{i}$ values are more outlying than $X$. Therefore, assuming that $X \sim F$, then a small value of $r_{G m}(X)$ suggests a possible deviation from $G$ to $F$. This may be due to a shifting in the location and/or an increase in the scale of the studied CTQ variable. Taking into account that the UCL is not defined for the $r$ chart, the CL line serves as a reference to identify emerging patterns, runs, or trends. If $r_{G m}(X)$ is greater than 0.5, there is evidence of scale decreasing, and also could take place a negligible location shift. This case should be tackled as an improvement in quality given a gain in the accuracy, and thus the process should not be considered as out of control.

\section{The $Q$ chart}

The idea behind the $Q$ chart is similar to the one behind the $\bar{x}$ chart. If $X_{1}, X_{2}, \ldots$ are univariate and $G$ is a normal distribution, the $\bar{x}$ chart plots the averages of consecutive subsets of the different $X_{i}$. A goal of this type of chart is that it can prevent the identification of a false alarm when the process is actually in control (even when some individual sample points fall out of control limits due to random fluctuations).

The $Q$ chart is the nonparametric alternative to the $\bar{x}$ chart. It is performed by plotting the averages of consecutive subsets of size $n$ corresponding to the rank statistic $\left(r_{G}\left(X_{i}\right)\right.$ or $\left.r_{G m}\left(X_{i}\right)\right)$, given by $Q\left(G, F_{n}^{j}\right)$ or $Q\left(G_{m}, F_{n}^{j}\right)$, whereas $F_{n}^{j}$ is the empirical distribution of the $X_{i}$ 's in the $j$ th subset, $j=1,2, \ldots$ Accordingly, if only $\left\{Y_{1}, Y_{2}, \ldots, Y_{m}\right\}$ are available, the $Q$ chart plots the sequence $\left\{Q\left(G_{m}, F_{n}^{j}\right), Q\left(G_{m}, F_{n}^{j}\right), \ldots\right\}$.

Depending on the value of $n$, the corresponding control limits are as follows:

- If $n \geq 5, C L=0.5$ and

$$
\begin{aligned}
& -L C L=0.5-Z_{\alpha}(12 n)^{\frac{1}{2}} \text { for } Q\left(G, F_{n}^{j}\right) . \\
& -L C L=0.5-Z_{\alpha} \sqrt{\frac{1}{12}\left(\frac{1}{m}+\frac{1}{n}\right)} \text { for } Q\left(G_{m}, F_{n}^{j}\right) .
\end{aligned}
$$

- If $n<5, C L=0.5$ and $L C L=\frac{(n ! \alpha)^{\frac{1}{n}}}{n}$.

\section{The $S$ control chart}

The $S$ control chart is based on the CUSUM univariate control chart, which is basically the plot of $\sum_{i=1}^{n}(X-\mu)$, which reflects the pattern of the total deviation from the expected value. As mentioned above, it is more effective than the $X$ chart or the $\bar{x}$ chart in detecting small process changes. The nonparametric CUSUM chart based on data depth suggests plotting $S_{n}(G)$ and $S_{n}\left(G_{m}\right)$, defined by

$$
S_{n}(G)=\sum_{i=1}^{n}\left(r_{G}\left(X_{i}\right)-\frac{1}{2}\right)
$$

with control limits $C L=0$ and $L C L=-Z_{\alpha}\left(\frac{n}{12}\right)^{\frac{1}{2}}$ and

$$
S_{n}\left(G_{m}\right)=\sum_{i=1}^{n}\left(r_{G_{m}}\left(X_{i}\right)-\frac{1}{2}\right) \text {. }
$$

If only $Y_{1}, \ldots Y_{m}$ are available, the control limits are $C L=0$ and $L C L=-Z_{\alpha} \sqrt{n^{2} \frac{\left(\frac{1}{m}+\frac{1}{n}\right)}{12}}$. The LCL control limits in both cases constitute a curve instead of a straight line; if $n$ is large, the control chart $S$ should be standardized as follows:

$$
\begin{gathered}
S_{n}^{*}(G)=\frac{S_{n}^{*}(G)}{\sqrt{\frac{n}{12}}} \\
S_{n}^{*}\left(G_{m}\right)=\frac{S_{n}\left(G_{m}\right)}{\sqrt{n^{2} \frac{\left(\frac{1}{m}+\frac{1}{n}\right)}{12}}}
\end{gathered}
$$

Therefore, this $S^{*}$ chart is defined by $C L=0$ and $L C L=-Z_{\alpha}$. 


\section{Examples of $r, Q$ and $S$ control charts applied using synthetic data}

A bivariate data set is used to illustrate how the previously discussed control charts arise. In fact, a synthetic dataset composed of 540 observations of a bidimensional standard Gaussian variable has been simulated, in addition to 40 individuals corresponding to another bidimensional Gaussian variable with mean and standard deviation equal to 2 .

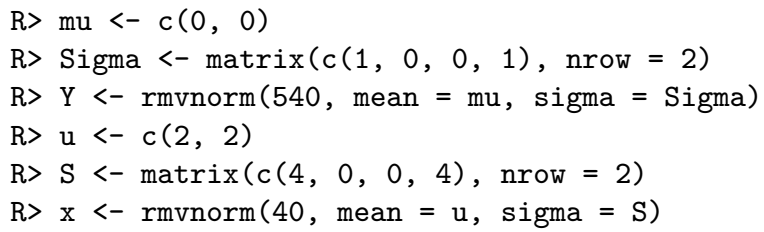

Prior to the application of nonparametric control charts, the dataset has to be converted into a npqcsd object. The synthetic dataset is arranged as two matrices, G composed of the 500 first rows (multivariate observations) of $\mathrm{Y}$, and $\mathrm{x}$ with the remaining ones and including those belonging to the second bidimensional variable

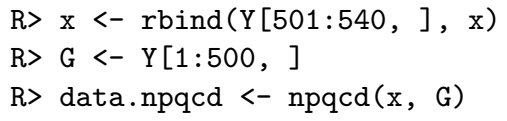

In the same way, the npqcd function creates a data object for non parametric quality control, the npqcs.r(), npqcs.Q(), and npqcs.S() functions computes all the statistics required to obtain the $r$, $Q$, and $S$ control charts, respectively. The argument method = c("Tukey", "Liu" , "Mahalanobis", "RP" , "LD") specifies the data depth function, and alpha is the signification level that defines the LCL. See Flores et al. (2021) to obtain additional information about these functions and their arguments.

\section{$r$ chart}

The $r$ control chart can be obtained by applying the npqcs.r() function to the npqcd object and plotting the result.

$\mathrm{R}>$ res.npqcs <- npqcs.r (data.npqcd, method $=$ "Tukey", alpha $=0.025$ )

$\mathrm{R}>$ plot(res.npqcs, title = " r Control Chart")

The resulting chart is shown in Figure 4, where it can be observed that the process is out of control from the 42nd observation, as expected, taking into account that most of the $r_{G m}\left(X_{i}\right)$ values are falling below the LCL.

\section{$Q$ chart}

In this case, the dataset is assumed to be composed of rational samples of size 4 . Thus, the $Q$ nonparametric alternative of $\bar{x}$ chart is proposed and applied to control the bidimensional process:

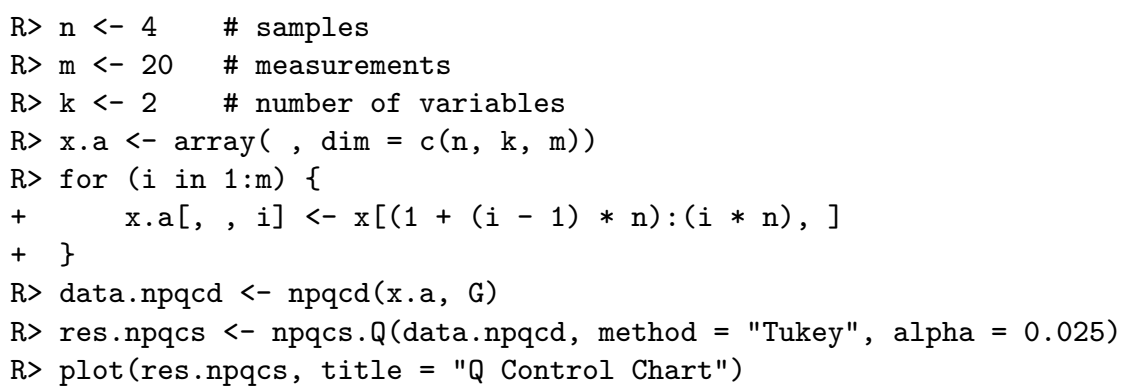

Figure 5 clearly shows that the process is out of control in the second half, from the 20th rational sample. We can also see that the high random fluctuations of the $r$ chart are attenuated in the $Q$ chart due to the averaging effect.

\section{$S$ chart}

Finally, the nonparametric counterpart of CUSUM control chart is performed from the multivariate individual observations. 


\section{r Control Chart}

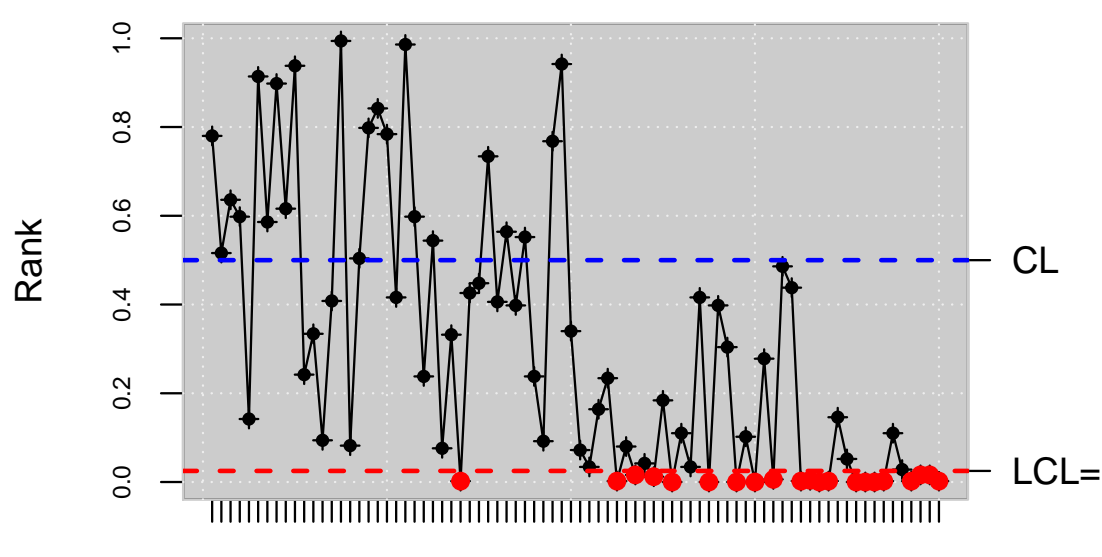

Sample

Figure 4: $r$ control chart.

\section{Q Control Chart}

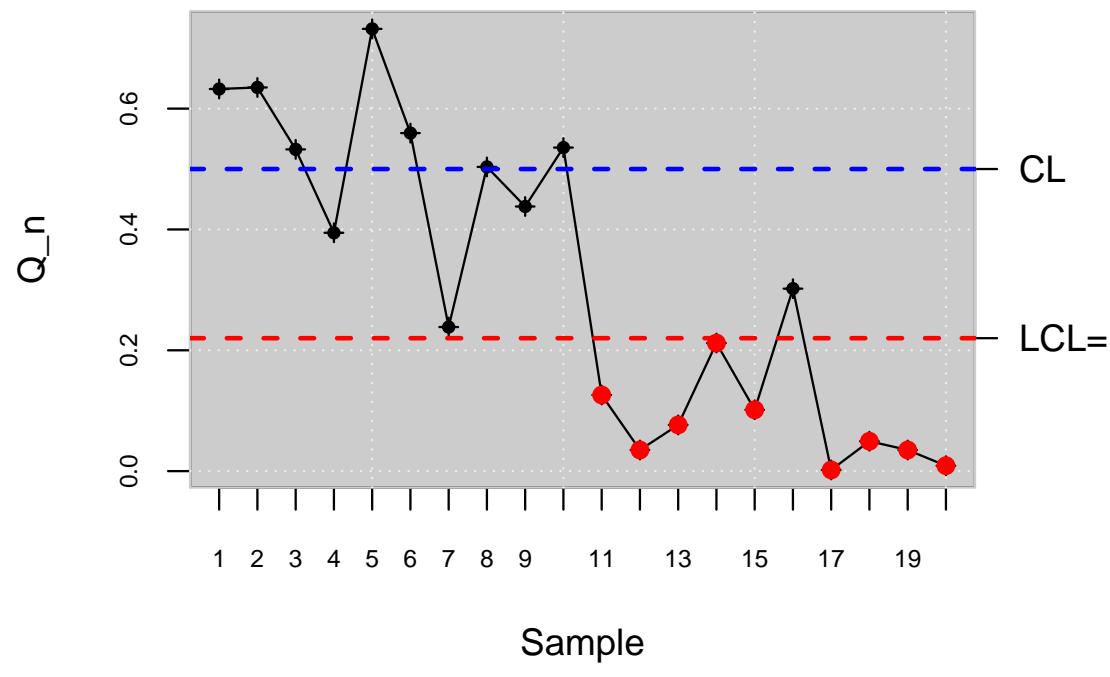

Figure 5: $Q$ control chart.

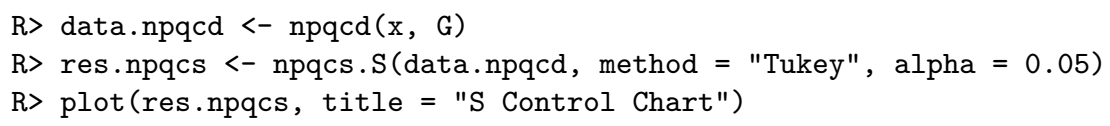

Figure 6 shows that the process is out of control from the 48th observation. Note that the $S$ graph performs better in identifying small changes in a process. In this case, the performance of the $Q$ chart is better than the corresponding to the $S$ chart. 


\section{S Control Chart}

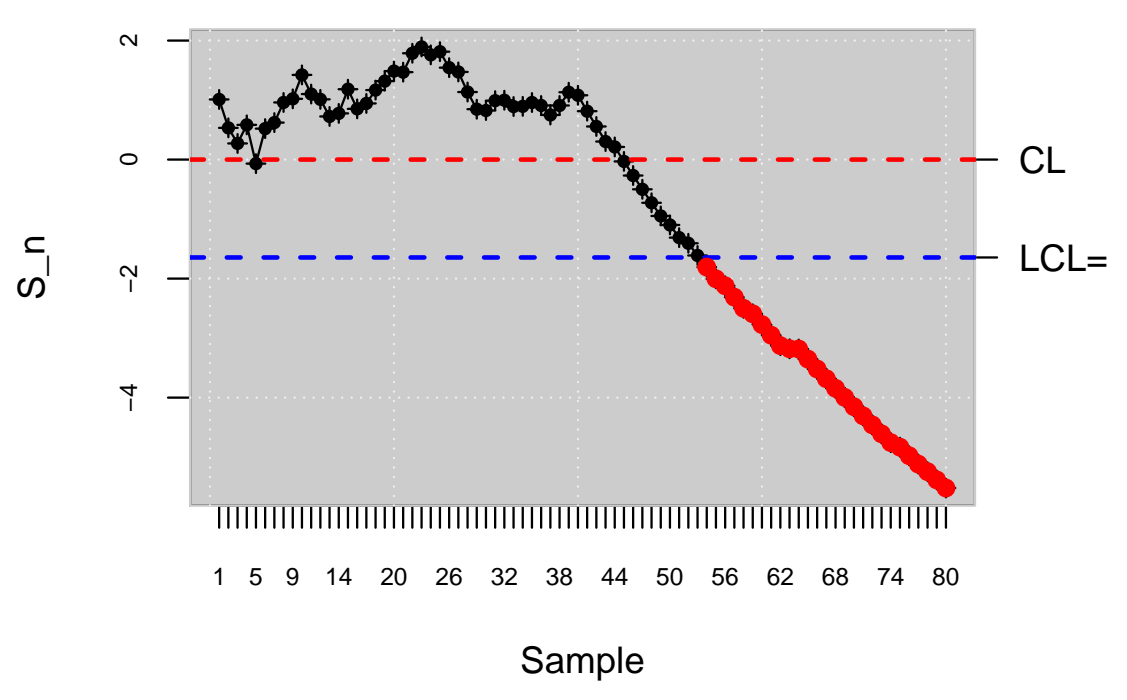

Figure 6: $S$ control chart.

\section{Control charts for functional data based on data depth}

In the paradigm of Industry 4.0, processes and services are many times described by continuously monitored data of hourly, daily, monthly curves or smooth functions. When the processes are defined by functional data, the authors encourage to apply control charts based on Functional Data Analysis (FDA) in order to implement control and improvement tasks. In this section, the use of control charts presented in Flores et al. (Flores et al., 2020). Summarizing, this methodology consists of the proposal of new Phase I and Phase II control charts to be applied in those case study in which the datum unit is a curve. Phase I control chart is based on the computation of functional data depth (specifically Fraiman and Muniz (Fraiman and Muniz, 2001), Mode (Cuevas et al., 2007), and random projections (Cuevas et al., 2007) data depth) from which a data depth control chart is developed. Once the in-control calibration sample is obtained, the Phase II control chart based on functional data depth and rank nonparametric control chart can be applied. In addition to the Phase I functional data depth and Phase II rank control charts, plots of functional envelopes from the original curves are provided in order to help to identify the possible assignable causes of out of control states.

\section{Estimating a Phase I control chart for functional data (calibration)}

A dataset is simulated in order to illustrate the use of FDA control charts for Phase I and II. A functional mean, $m u_{0}$, and a functional standard deviation, sigma, are defined as shown in (Flores et al., 2020). An $n_{0}=100$ hundred curves composed of $m=30$ points are simulated. They account for the calibration or retrospective sample.

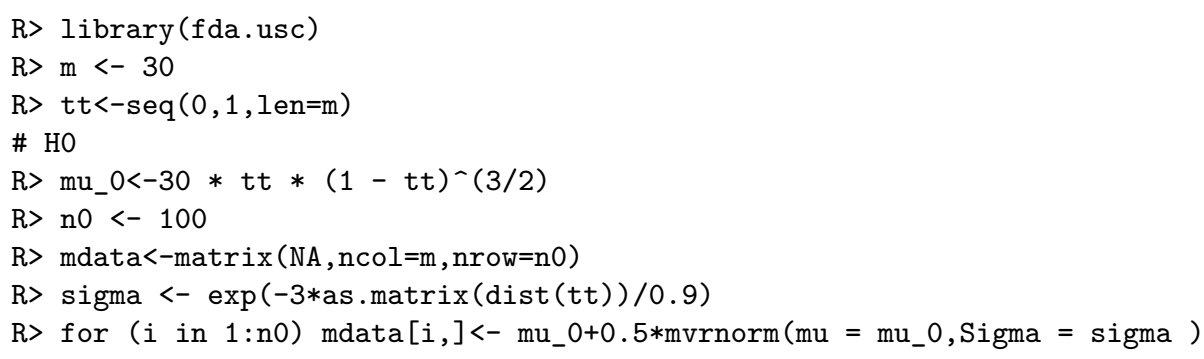

Prior to the application of control charts, the dataset is converted in a specific format by the fdqcd function. A plot function is also programmed to properly show the original functional data, plot.fdqcd. Figure 7 shows the original functional data consisting of curves. 
$R>$ fdchart <- fdqcd(mdata)

R> plot (fdchart, type="l", col="gray", main="Functional data")

\section{Functional data}

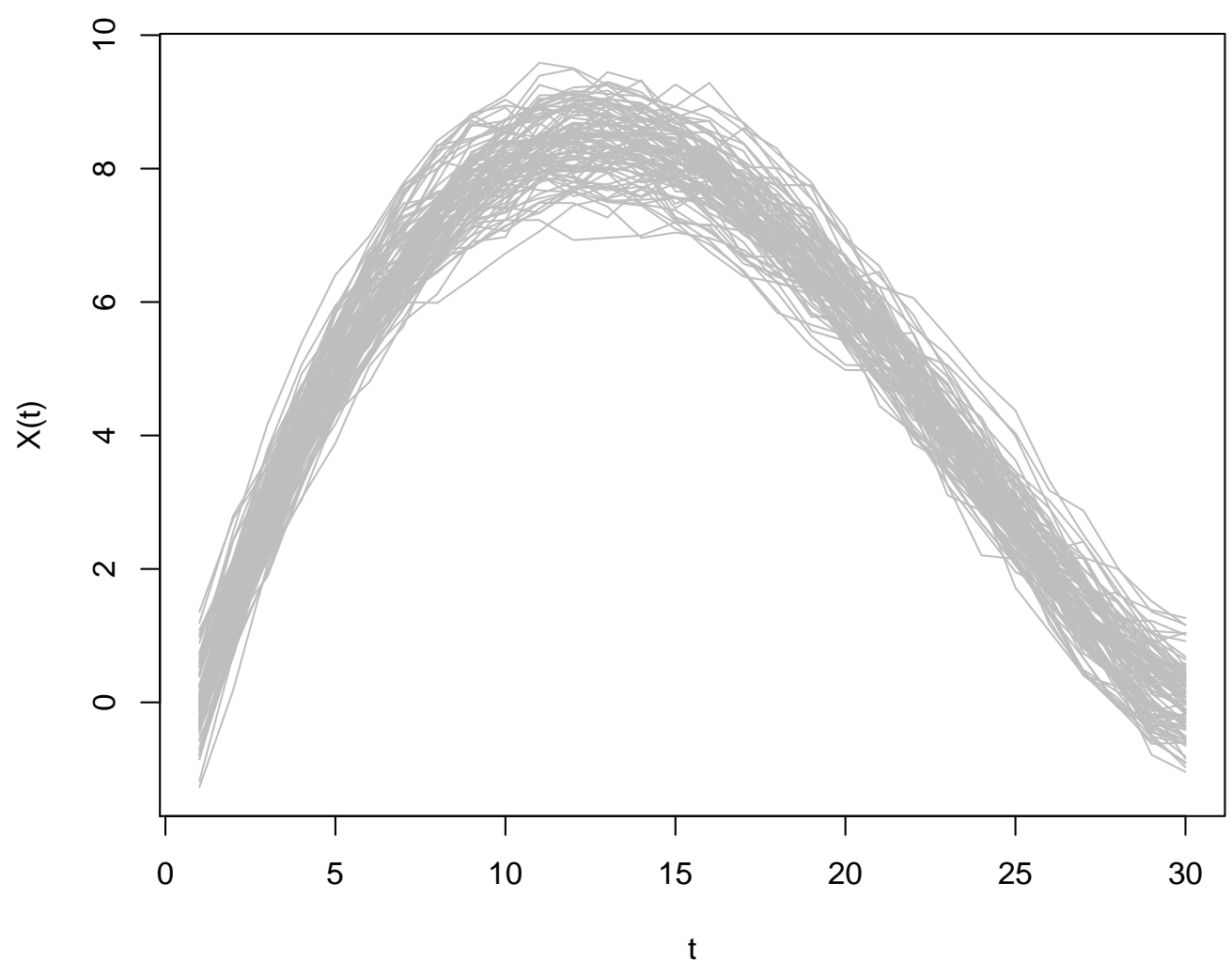

Figure 7: Original curves that account for the calibration sample.

The following step is to identify those curves that account for the in-control process. This task is done by the application of a Phase I control chart for functional data. This method is implemented in the qcr package by the fdqcs.depth function. Specifically, the arguments and default values for these functions are

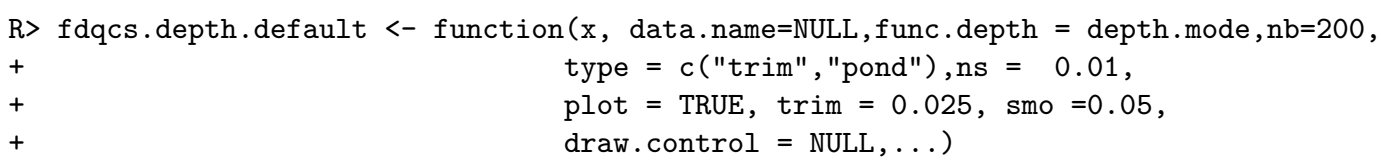

where func.depth is the type of depth measure, by default depth.mode, nb the number of bootstrap resamples, type accounts for the method used to trim the data, trim or pond (Flores et al., 2020), ns is the quantile to determine the cutoff from the bootstrap procedure (Flores et al., 2020), plot a logical value indicating that it should be plotted, trim the percentage of the trimming, smo the smoothing parameter for the bootstrap resampling (Flores et al., 2020), whereas draw.control specifies the col, Ity, and lwd for the fdataobj, statistic, IN and OUT objects. When the fdqcs.depth function is applied to the curves of the calibration sample, the fddep object of fdqcs. depth class is obtained. It is composed of the original data, the depth corresponding to each curve, the lower control limit of the depth chart, the index of those curves out of control, the curves that account for the limits of the envelope composed by the deepest cures, and the deepest curve or functional median.

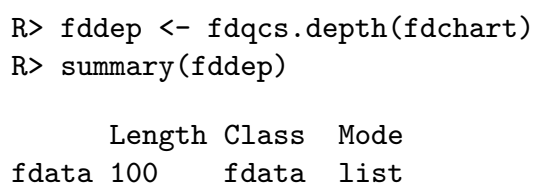




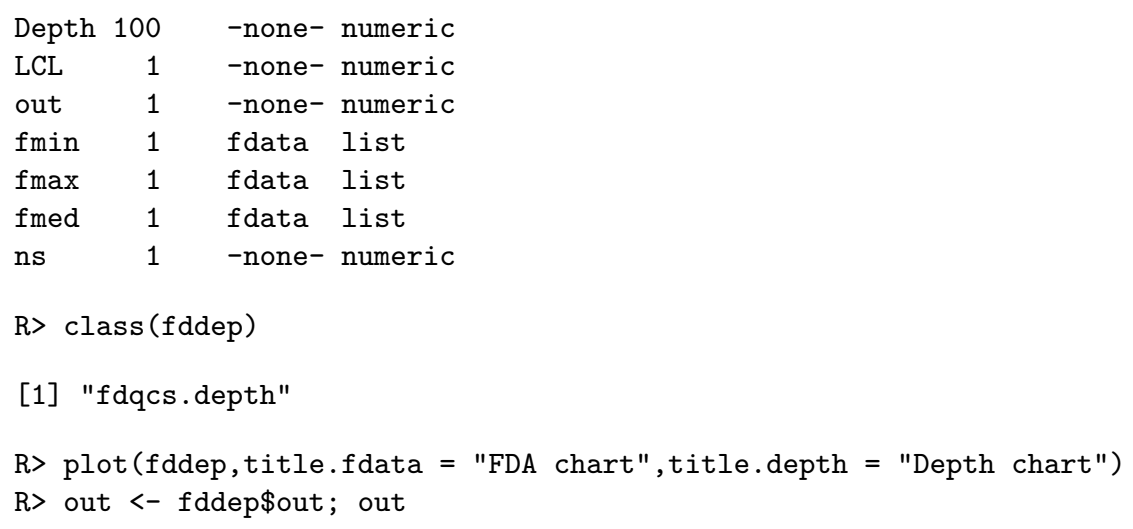

\section{[1] 29}

Figure 8 shows the control chart for the depth of the curves (right panel). The LCL is estimated by a smoothed bootstrap procedure (Flores et al., 2020). In order to provide a tool to identify the assignable cause of each out-of-control curve, the original curves with the envelope with the $99 \%$ of the deepest curves are also shown (left panel). The analysis of the shape and magnitude of the curves in and out of bounds can help to associate each curve out of control to an assignable cause, allowing for processes control, maintenance, and improvement.
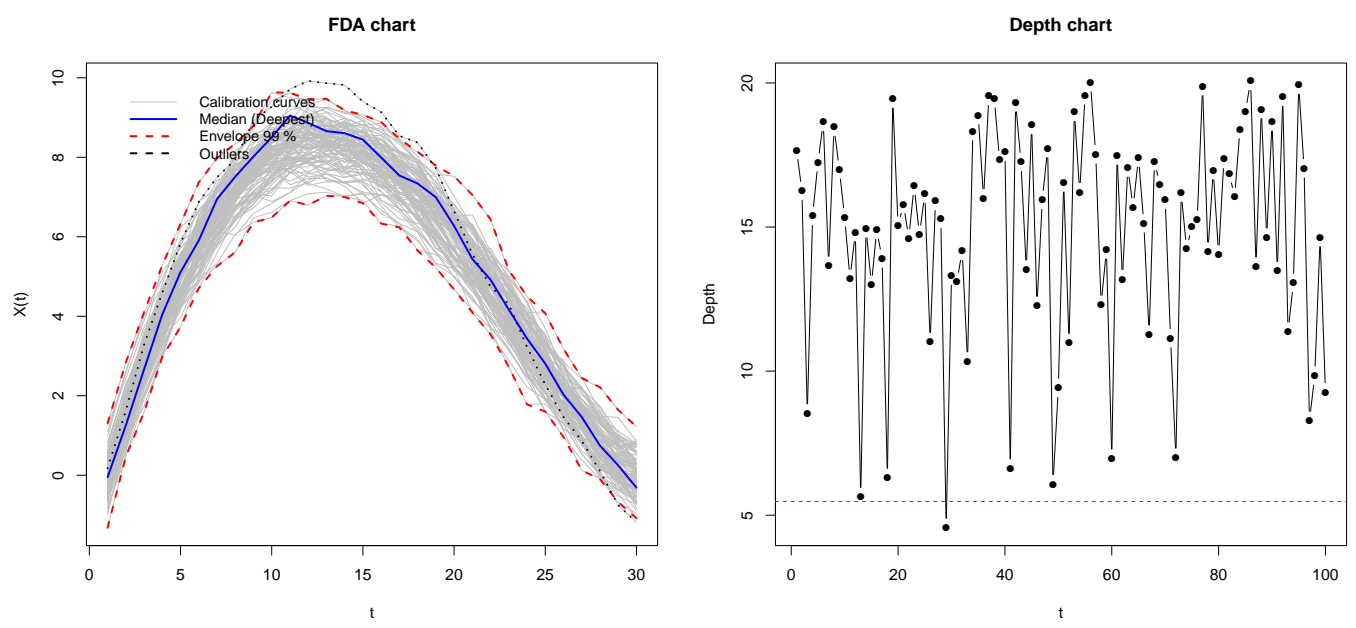

Figure 8: Left panel: Original curves with the envelope composed of $90 \%$ of the deepest curves. Right panel: Control chart for the depths of the curves (the LCL has been estimated by bootstrap procedures at a signification level of $10 \%$.

Phase I ends when a calibration sample without curves out of control is obtained. The iterative procedure to obtain an in control calibration sample is shown in the following lines.

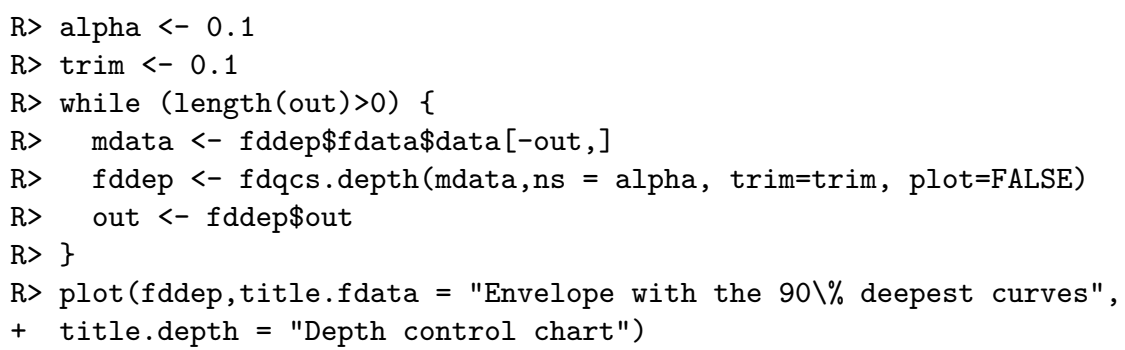

Figure 9 is obtained from the application of plot function to fddep object. It shows that all the curves of the calibration sample are in control, and thus, the natural variability of the process is estimated. 

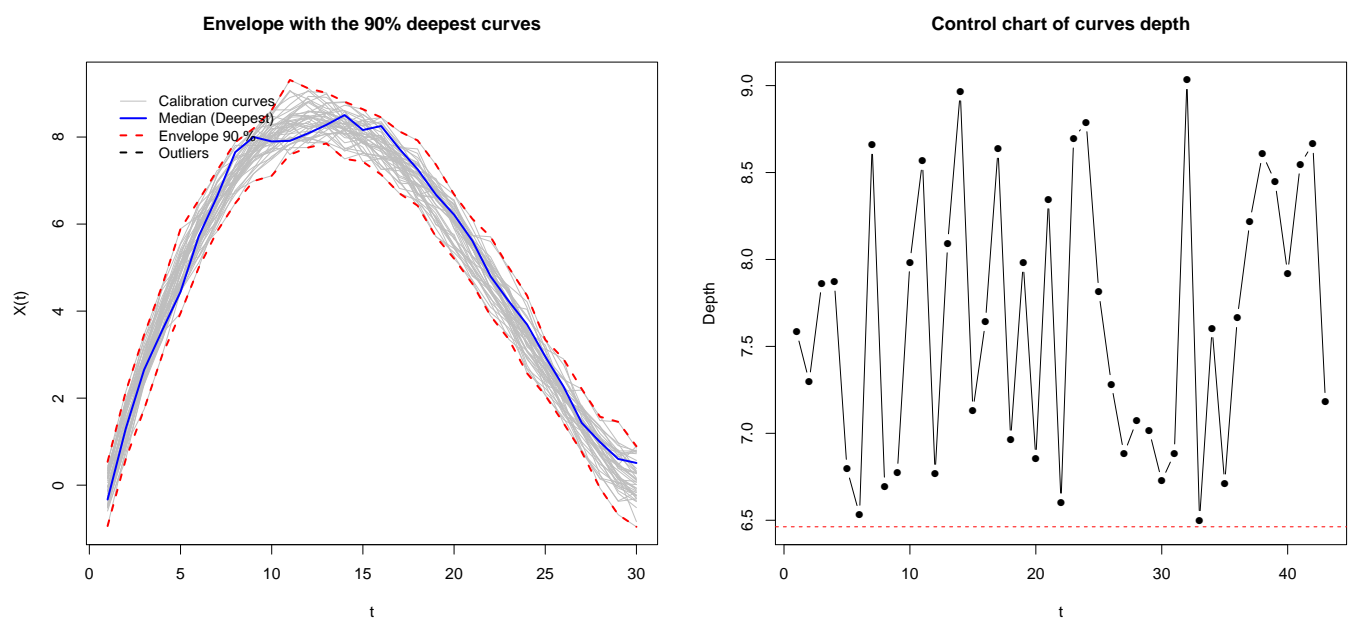

Figure 9: Results corresponding to the second iteration to obtain the in control calibration sample. Left panel: Original curves with the envelope composed of the $90 \%$ of the deepest curves. Right panel: Control chart for the depths of the curves (the LCL has been estimated by bootstrap procedures at a signification level of $10 \%$.

\section{Estimating a Phase II control chart for functional data (monitoring)}

The next step is to perform the Phase II of process control. The monitoring phase is performed by the application of Phase II control charts for functional data based on multivariate nonparametric control charts. Firstly, a monitoring sample composed of 50 curves is simulated by the following code.

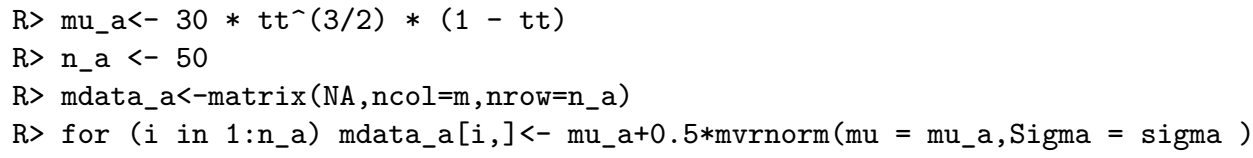

The curves of the monitoring sample are defined with fdqcd format and a control chart for Phase II is developed by applying the fdqcs.rank function. It is composed by the following arguments, fdqcs.rank $(\mathrm{x}, \mathrm{y}=\mathrm{x}$, func.depth $=$ depth.FM, alpha $=0.01$, plot $=$ TRUE, trim $=0.1$, draw . control $=$ NULL,$\ldots)$.

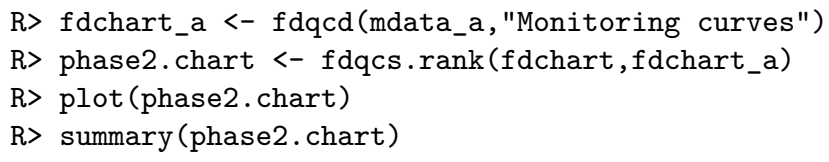

Figure 10 accounts for the FDA chart with the calibration sample and its envelope composed by the deepest curves. Moreover, the monitoring sample is also included and compared with the calibration sample by using the FDA chart. In addition, the Phase II rank control chart for functional data is shown including both calibration and monitoring samples or only the ranks corresponding to the monitoring sample. The second population that corresponds with the monitoring sample is identified by the control chart from the first monitored curve (panels below in Figure 10).

\section{Process capability analysis}

The analysis of the capability of a process in the case of statistical quality control is done through the calculation of the so-called capability. These indices measure whether a process is capable or not of meeting the corresponding technical specifications set by the customer, or the manufacturer, by comparing those with the natural variability of the CTQ variable that characterizes the process. The interpretation of these indices is associated with the result of this relation. Capability indices are generally calculated as the ratio between the length of the specification interval and the natural variability of the process in terms of $\sigma$. Large values of these indices mean that the corresponding process is capable of producing articles that meet the requirements of the client and manufacturers. 
Fdata Chart

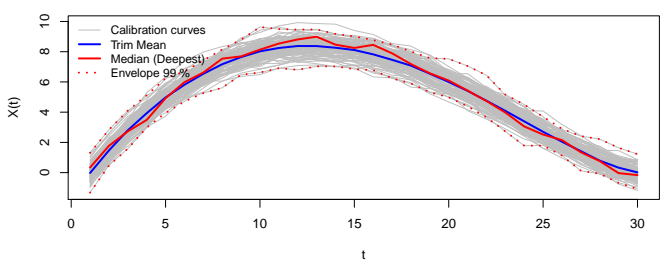

Phase II: Rank Chart

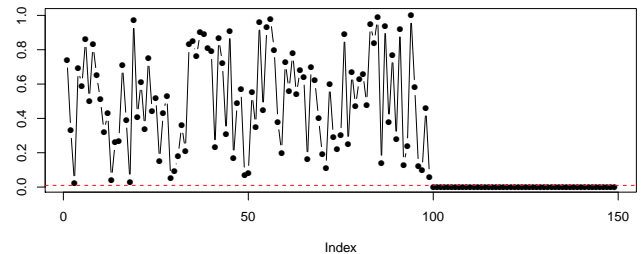

Fdata Chart

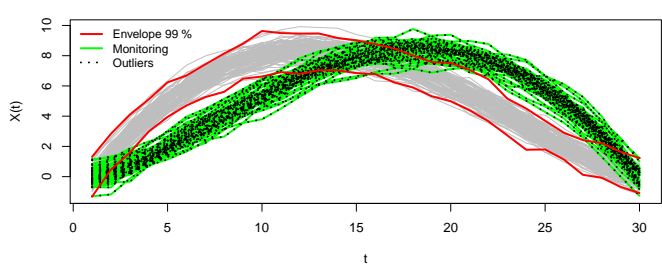

Phase II: Rank Chart

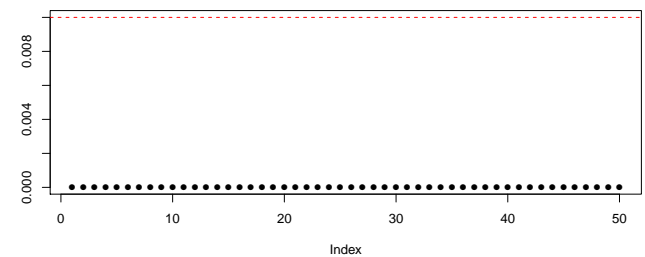

Figure 10: First row: the left and right panels show the FDA charts for the calibrating and monitoring samples with a signification level of 0.01. Second row: The left and right panels show the Phase II rank control chart for functional data, including calibration and monitoring sample (left panel) and only monitoring sample (right panel).

In other words, the larger the value of the capability index, the smaller the number of products outside the specification limits.

In this section, we describe the capability indices for processes whose underlying distribution is normal and not normal (exponential, Weibull, etc.). However, it is important to note that the development of programming tools for nonparametric capability analysis is one of the main goals and contributions of the qcr package. In addition to the estimation of capability indices, a graphical output is provided. Based on the proposal of qualityTools package, the qcr graphical output for capability analysis includes a normality test for the CTQ variable, a Q-Q plot, a histogram with the theoretical Gaussian distribution density, parametric and nonparametric estimates of capability indices and a contour capability control chart. In the following lines, parametric and nonparametric capability analysis utilities are described using different examples of applications.

\section{Assuming a normal distribution}

The most widely used capability indexes in the industry analyze the process capability under the assumptions of a stabilized process (in control) and a Gaussian distributed CTQ variable. Table 3 shows the main parametric (assuming Gaussian distribution) indices, namely $C_{p}, C_{p k}, C_{p m}$, and $C_{p m k}$.

Vännman (1995) proposed a general formulation of these indices by an expression that depends on the non-negative parameters $u$ and $v$ :

$$
C_{p}(u, v)=\frac{d-u|\mu-m|}{3 \sqrt{\sigma^{2}+v(\mu-T)^{2}}},
$$

whereby $d=(U S L-L S L) / 2, m=(L S L+U S L) / 2, U S L$ is the upper specification limit, the $L S L$ is the lower specification limit, $\sigma$ is the theoretical standard deviation, $\mu$ accounts for the theoretical mean of the CTQ variable, and $T$ is the specification target (by default the mean between the $L S L$ and $U S L)$. The indices shown in Table 3 are obtained from this expression just considering values of 0 or 1 for $u$ and $v: C_{p}(0,0)=C_{p}, C_{p}(1,0)=C_{p k}, C_{p}(0,1)=C_{p m}, C_{p}(1,1)=C_{p m k}$.

The piston rings data set is used to illustrate the calculation of the capability indices using the qcs.cp () function based on the expressions previously described in Table 3. From the statistics obtained from the $\bar{x}$ control chart of pistonrings dataset, the $\gamma$ and $\beta$ values are estimated, and the corresponding capability index is computed.

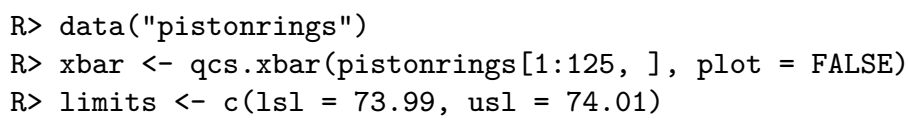




\begin{tabular}{|l|l|}
\hline Potential capability & $\hat{C}_{p}=\frac{U S L-L S L}{6 \hat{\sigma}}$ \\
\hline $\begin{array}{l}\text { Actual capability with respect } \\
\text { to the specification limits }\end{array}$ & $\hat{C}_{p, \text { lower }}=\frac{\hat{\mu}-L S L}{3 \hat{\sigma}}$ \\
$\hat{C}_{p, \text { upper }}=\frac{U S L-\hat{\mu}}{3 \hat{\sigma}}$ \\
$\begin{array}{l}\text { Shifting of the mean with } \\
\text { respect to the target }\end{array}$ & $\hat{C}_{p k}=\min \left[\frac{U S L-\hat{\mu}}{3 \hat{\sigma}}, \frac{\hat{\mu}-L S L}{3 \hat{\sigma}}\right]$ \\
\hline $\begin{array}{l}C_{p k} \text { correction for detecting } \\
\text { deviations with respect to the target }\end{array}$ & $\hat{C}_{p k m}=\frac{\hat{C}_{p}}{\sqrt{1+\left(\frac{\hat{\mu}-T}{\hat{\sigma}}\right)^{2}}}$ \\
\hline
\end{tabular}

Table 3: PCR from first to fourth generation, $U S L$ is the upper specification limit, $L S L$ is the lower specification limit, $\mu$ is the real mean, $\hat{\mu}$ is the estimated mean, and $\hat{\sigma}$ is the estimated standard deviation.

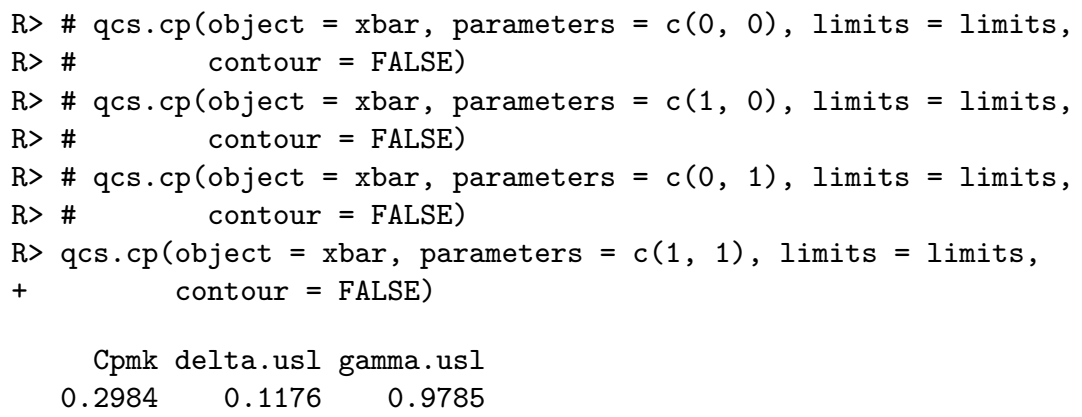

Consequently, the obtained results are $C_{p}=0.3407, C_{p k}=0.3006, C_{p m}=0.3382$, and $C_{p m k}=0.2984$, respectively. The argument parameters account for $u$ and $v$ values, while object is the type of control chart from which the $\sigma$ is estimated, limits are the specification control limits, and contour is the parameter that indicates when the process capability contour chart is plotted.

\section{Process capability plot}

In Vännman (2001) and Deleryd and Vännman (1999), a graphical method (based on common capability indices) to analyze the capability of a process is proposed. The goal of using this type of plot (if compared with respect to only capability indices calculation) is to provide immediate information of the location and spread of the CTQ feature and about the capability to meet the specifications of the corresponding process. When using this chart, a process will be capable if the process capability index is higher than a certain value $k$, with $k>1$. The most used values for $k$ are $k=1, k=4 / 3$, or $k=5 / 3$, even 2 at a Six Sigma level, taking into account the usual index limits for which a process could be assumed capable. It will also be assumed that the target value matches the center of the specification interval, that is, $T=\frac{(U S L+L S L)}{2}=m$. Then, one of the indices defined by the $C_{p}(u, v)$ family is used, e.g., $C_{p k}$ or $C_{p m}$, and the process will be defined as capable if $C_{p}(u, v)>k$, given the values of $u, v$, and $k$. Also note that if $\mu=T$, all the $C_{p}(u, v)$ indices are defined by the same expression as the $C_{p}$. Moreover, different setting for $u, v$, and $k$ impose different constraints on the process parameters $(\mu, \sigma)$. This can be easily seen through a process capability plot. This graph is a contour plot of $C_{p}(u, v)=k$ as a function of $\mu$ and $\sigma$, but it can also be defined as a function of $\delta$ and $\gamma$, with $\delta=\frac{\mu-T}{d}$ and $\gamma=\frac{\sigma}{d}$. The contour line is obtained by rewriting the index $C_{p}(u, v)$ as a function of $\delta$ and $\gamma$ as follows $C_{p}(u, v)=\frac{1-u|\delta|}{3 \sqrt{\gamma^{2}+v(\delta)^{2}}}$. Therefore, the $C_{p}(u, v)=k$ equation is solved, plotting $\gamma$ depending on the values of $\delta$. The resulting expressions are:

$$
\gamma=\sqrt{\frac{(1-u|\delta|)}{9 k^{2}}-v \delta^{2}},|\delta| \leq \frac{1}{u+3 k \sqrt{v}},(u, v) \neq(0,0)
$$

When $u=v=0$, that is, when we consider the index $C_{p}=k$, we have $\gamma=\frac{1}{3 k}$ and $|\delta| \leq 1$. It is important to highlight that the $\gamma$-axis accounts for the process spread, whereas the $\delta$-axis accounts for the process location. The values of the parameters $\mu$ and $\sigma$ which provide values $(\delta, \gamma)$ within the region bounded by the contour line $C_{p}(u, v)=k$ and the $\delta$-axis will provide a larger $C_{p}(u, v)$ 
value than $k$, leading a capable process. Furthermore, values of $\mu$ and $\sigma$ which provide values $(\delta, \gamma)$ outside this region will provide a value $C_{p}(u, v)$ smaller than $k$, i.e., a non-capable process. In the case of the process not being capable, this type of plot is useful to understand if the corrective actions have to be performed to decrease the process spread, or the process location (deviation with respect to target), or even when both changes are needed to improve the process capability. This can be observed by observing the distance with respect to the $x$ and $y$-axis. Below are some examples of capability plot application which can be generated through the application of the qcs.cp function with contour=TRUE and $\mathrm{k}=1$ (default values):

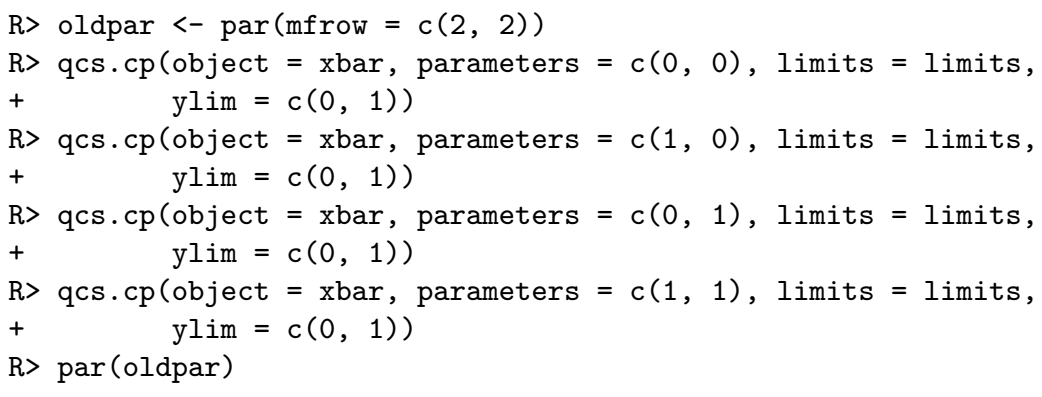

The result is shown in Figure 11. In all the cases, the points in red are out of the area defined by the line in blue and the $\delta$ axis. Thus, the corresponding process is not capable, no matter the capability index that is used. In any case, note that the $C_{p}$ index is useless in identifying non-capable processes due to location shifts with respect to the target. In the same way, the $C_{p k}$ index assumes as capable processes that are far from the target as long as they were close to the specification limits (as shown in Figure 11). Thus, the use of the $C_{p m}$ and $C_{p m k}$ are recommended due to they take into account both shifts from the target and the spread. In the present case, the process is not capable due to the spread rather than the target shift. Therefore, the process changes could be due to decreases in the variability process.

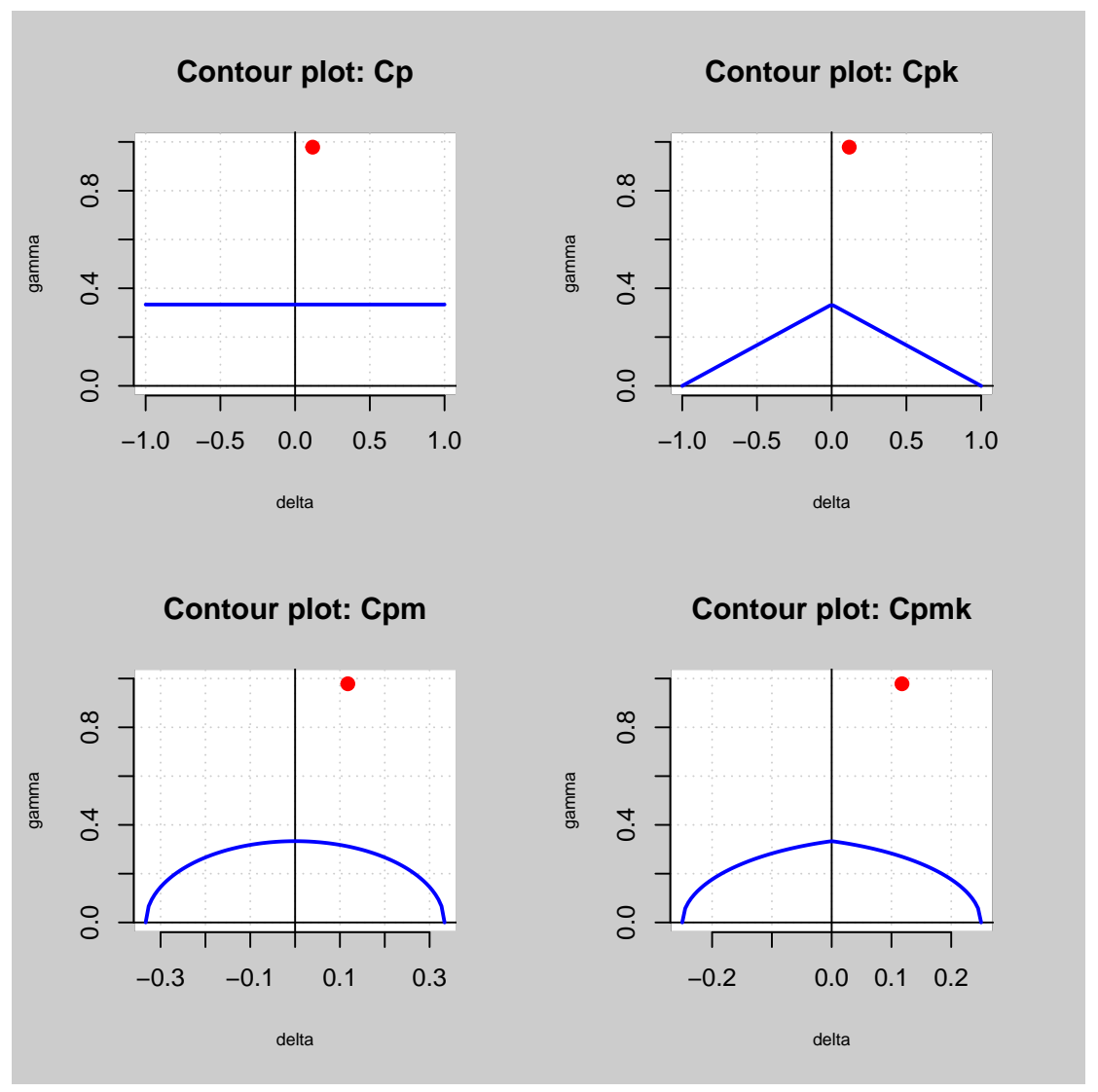

Figure 11: Process capability plots using the $C_{p}, C_{p k}, C_{p m}$, and $C_{p m k}$ indexes. 


\section{Estimated process capability plot}

In practice, the process parameters are unknown and we need to estimate them. We can perform a decision rule based on the sample statistics that provide a sample estimate of the capability index and, finally the so called estimated process capability plot, also called $\gamma^{*}-\delta^{*}$ plot (Deleryd and Vännman, 1999). It allows us to decide whether a process is capable or not assuming that $\mu$ and $\sigma$ parameters are unknown and estimated by $\hat{\mu}=\bar{X}$ and $\hat{\sigma}^{2}=\frac{1}{n} \sum_{i=1}^{n} X_{i}^{2}-\bar{X}^{2}$. They are the maximum likelihood estimators when the CTQ variable of the process is normally distributed, and $X_{1}, X_{2}, \ldots, X_{n}$ is a random sample of a normal distribution with $\mu$ mean and $\sigma^{2}$ variance.

The qcr package only provides the $\gamma^{*}-\delta^{*}$ plot corresponding to the $C_{p m}$ index taking into account that the other capability indices do not consider shifts from the target value in their calculations. For the general case, see the work of Vännman (2001). In order to obtain an appropriate decision rule for the case of $C_{p m}$ index, we test the hypotheses $H_{0}: C_{p m} \leq k_{0}$ versus $H_{1}: C_{p m}>k_{0}$, using

$$
\hat{C}_{p m}=\frac{d}{3 \sqrt{\hat{\sigma}^{2}+(\hat{\mu}-T)^{2}}}
$$

as test statistic. The null hypothesis will be rejected if $\hat{C}_{p m}>c_{\alpha}$, where the constant $c_{\alpha}$ is determined by previously defining a signifition test level $\alpha$. Vännman (2001) showed that the null hypothesis $H_{0}: C_{p m} \leq k_{0}$ can be reduced to $H_{0}: C_{p m}=k_{0}$. Thus, for given values of $\alpha$ and $n$, the process will be considered capable if $\hat{C}_{p m}>c_{\alpha}$, with $c_{\alpha}>k_{0}$. Hubele and Vannman (2004) proved that, when the $C_{p m}$ index is used, the critical value for a given $\alpha$ is obtained as

$$
c_{\alpha}=k_{0} \sqrt{\frac{n}{\chi_{\alpha, n}^{2}}},
$$

where $\chi_{\alpha, n}^{2}$ is the quantile $\alpha$ of a $\chi^{2}$ distribution with $n$ degrees of freedom. The qcr package includes the qcs.hat.cpm() function to obtain both the theoretical capability plot and the estimated capability plot from sample statistics. Among other options, the user can indicate the control chart from which the estimates $\hat{\mu}$ and $\hat{\sigma}$ are obtained (alternatively, $\hat{\mu}$ and $\hat{\sigma}$ can be introduced through mu and std.dev), and the specification limits using limits. Furthermore, the signification level and the capability limit can be modified, as they are set to $\alpha=0.05$ and $k_{0}=1$ by default. The following code illustrates its application to pistonrings data.

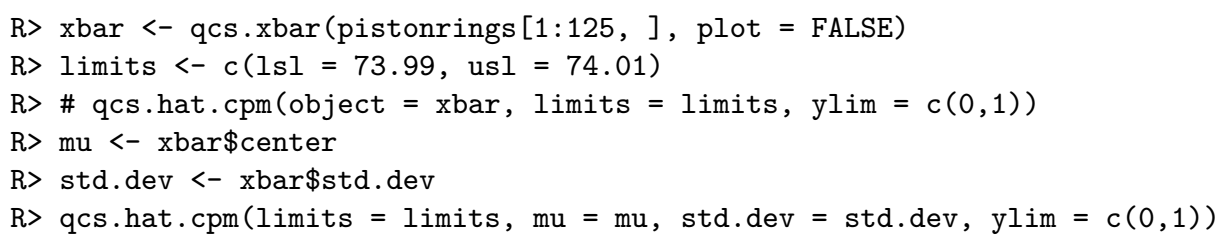

The result is shown in Figure 12. The contour line corresponding to the capability region obtained from the capability index sample is always more restrictive than the corresponding theoretical one.

\section{Nonparametric capability analysis}

Traditional assumptions about data such as normality or independence are frequently violated in many real situations. Thus, in scenarios in which assumptions of normality are not verified, the indices defined in the previous sections are not valid. Pearn and Chen (1997) and Tong and Chen (1998) proposed generalizations of $C_{p}(u, v)$ for the case of arbitrary distributions of data

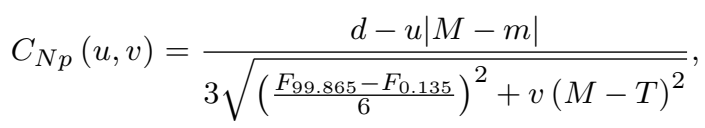

where $F_{\alpha}$ is the percentile $\alpha \%$ of the corresponding distribution and $M$ the median of the process. However, the distribution of the underlying process is always unknown. Chang and Lu (1994) calculated estimates for $F_{99.865}, F_{0.135}$ and $M$ based on the sample percentiles.

Pearn and Chen (1997) proposed the following estimator

$$
\hat{C}_{N p}(u, v)=\frac{d-u|\hat{M}-m|}{3 \sqrt{\left(\frac{U_{p}-L_{p}}{6}\right)^{2}+v(\hat{M}-T)^{2}}}
$$




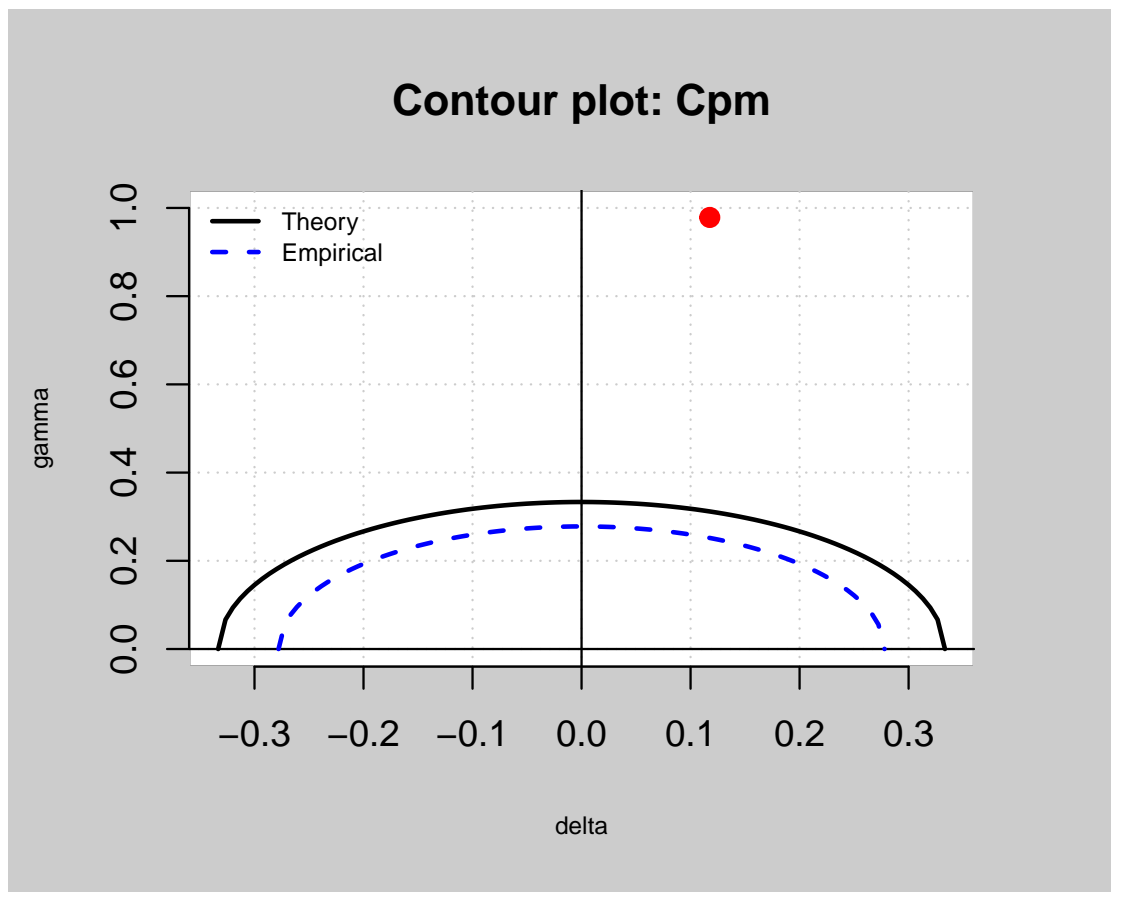

Figure 12: Comparison between theorical and estimated process capability plots.

where $U_{p}$ is an estimator for $F_{99.865}, L_{p}$ is an estimator for $F_{0.135}$, and $\hat{M}$ is an estimator for $M$, obtained from the tables developed by Gruska et al. (1989).

The qcs.cpn() function of qcr calculates $C_{N p}, C_{N p k}, C_{N p m}$, and $C_{N p m k}$ using the formulation described by Tong and Chen (1998). The code that illustrates its use is shown below. To obtain the nonparametric capability indices it is necessary to indicate the $u$ and $v$ parameters.

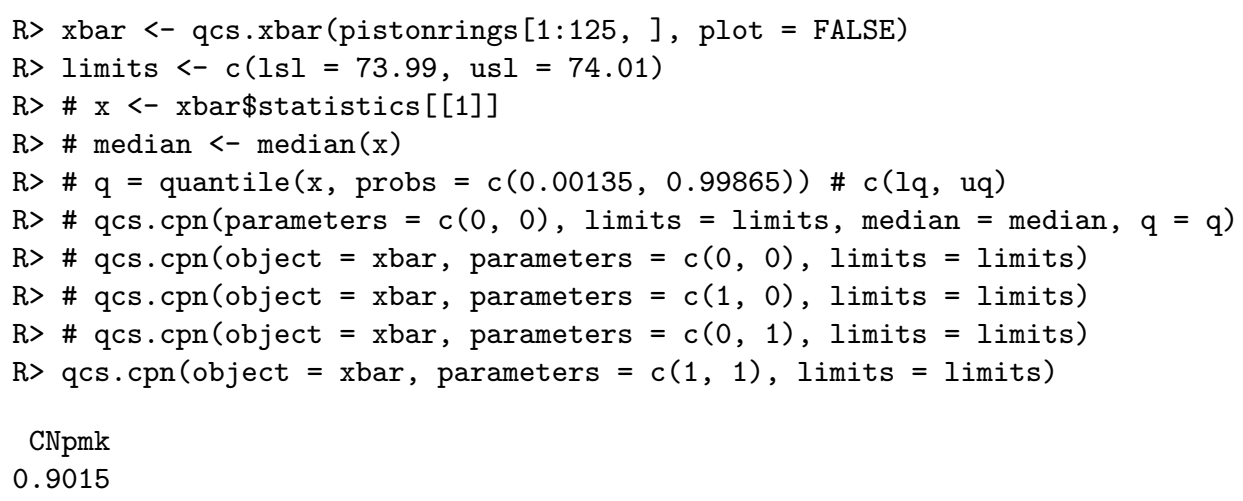

Thus, the values obtained are $C_{N p}=1.0082, C_{N p k}=0.9275, C_{N p m}=0.9799$ and $C_{N p m k}=0.9015$. If a capability limit of $k=1$ or $k=1.33$ is assumed, we can infer that the process is not actually capable to meet the customers or manager's requirements.

\section{Tools for a comprehensive processs capability analysis}

Function qcs.ca() provides a comprehensive information of the capability of a process, summarized through a graphical output. This function calculates the process capability indices $C_{p}, C_{p k}, C_{p L}$, $C_{p U}, C_{p m}, C_{p m k}$ from a qcs object, assuming a Gaussian distribution. Moreover, it computes confidence limits for $C_{p}$ using the method described by Chou et al. (1990). Approximate confidence limits for $C_{p l}, C_{p u}$, and $C_{p k}$ are also estimated using the method described in Bissell (1990), while the confidence limits for $C_{p m}$ are based on the approximated method of Boyles (1991) that assumes the target is the mean of the specification limits. Moreover, the $C_{N p}, C_{N p k}, C_{N p m}$, and $C_{N p m k}$ nonparametric capability indices are also obtained. There is also a specific box within the summary plot that shows the proportion of observations and expected observations under the Gaussian assumption out of the specification limits (nonconforming observations). Further, a histogram of the 
data sample is provided, in addition to the corresponding Gaussian density curves obtained from the sample estimates (one per standard deviation estimate procedure). They are displayed along with the specification limits, a quantile-quantile plot for the specified distribution, and a process capability plot obtained from the $C_{p m}$ index (both using theoretical and sample alternatives). In order to describe the qcs.ca() performance, the following code corresponds to the analysis of the first 125 observations of the pistonrings dataset (the corresponding output is shown in Figure 13).

R> qcs.ca(xbar, limits $=c(1 s l=73.99$, usl $=74.01))$

Process Capability Analysis

Call:

qcs. $c a($ object $=$ xbar, limits $=c(l s l=73.99$, usl $=74.01))$

Number of obs $=125 \quad$ Target $=74$

$\begin{array}{ll}\text { Center }=74 & \text { LSL }=73.99\end{array}$

StdDev $=0.009785 \quad$ USL $=74.01$

Paremetric Capability indices:

$\begin{array}{lrrr} & \text { Value } & 0.1 \% & 99.9 \% \\ \text { Cp } & 0.3407 & 0.2771 & 0.4065 \\ \text { Cp_1 } & 0.3807 & 0.2739 & 0.4875 \\ \text { Cp_u } & 0.3006 & 0.2021 & 0.3991 \\ \text { Cp_k } & 0.3006 & 0.1944 & 0.4068 \\ \text { Cpm } & 0.3382 & 0.2749 & 0.4038\end{array}$

Non parametric Capability indices:

$\begin{array}{lr} & \text { Value } \\ \text { CNp } & 1.0082 \\ \text { CNpK } & 0.9275 \\ \text { CNpm } & 0.9799 \\ \text { CNpmk } & 0.9015\end{array}$

PPM :

Exp<LSL $1.267 \mathrm{e}+07$

Exp $>$ USL $1.836 \mathrm{e}+07$

Exp Total $3.103 e+07$
Obs $<$ LSL 0

Obs $>$ USL $8 \mathrm{e}+05$

Obs Total $8 \mathrm{e}+05$

Test:

Anderson Darling Test for normal distribution

data: xbar

$\mathrm{A}=0.1399$, mean $=74.001, \mathrm{sd}=0.005, \mathrm{p}$-value $=0.9694$

alternative hypothesis: true distribution is not equal to normal 


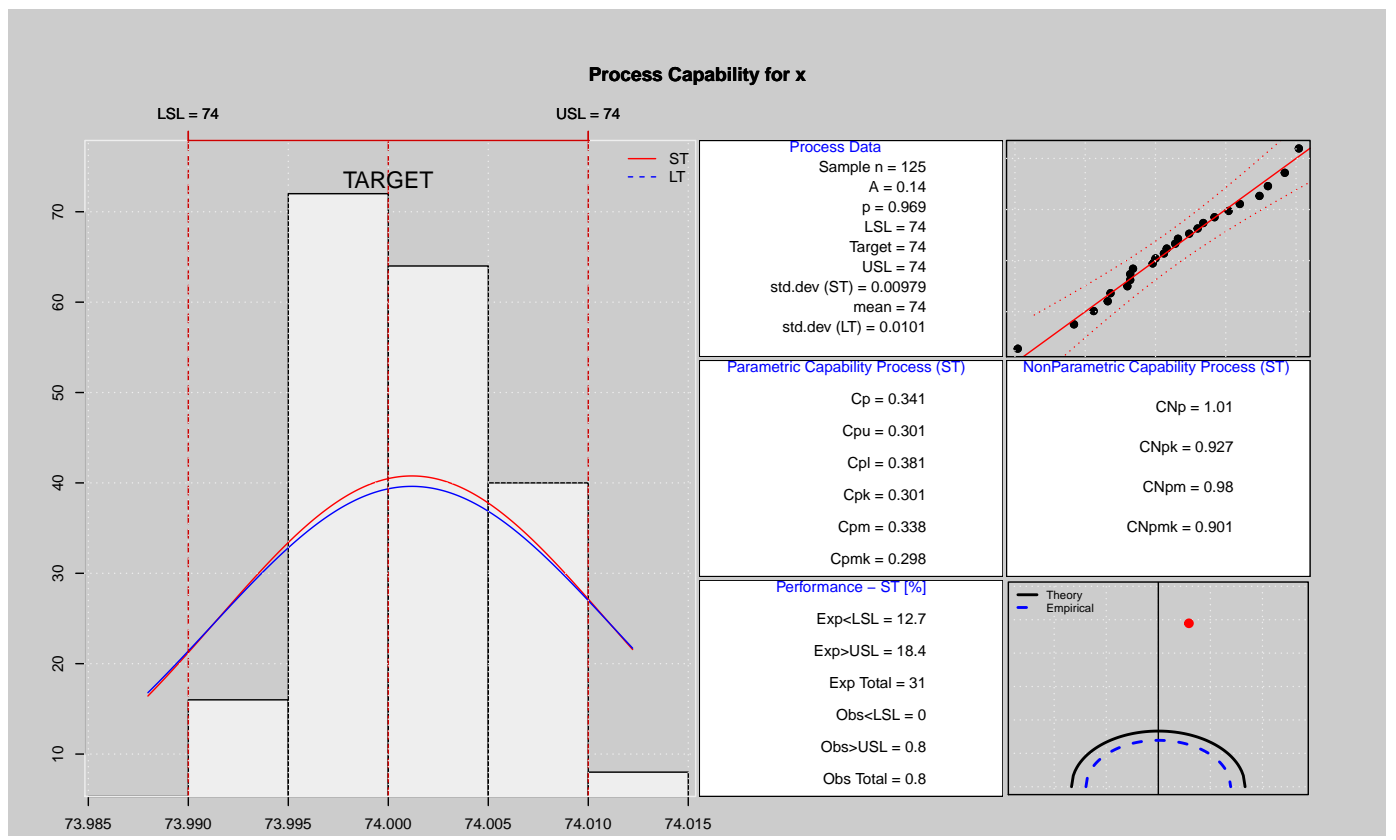

Figure 13: A complete analysis of the process capability.

\section{Conclusions}

The qcr package has been developed to provide users with a comprehensive set of functions that manage statistical process control, ranging from univariate parametric analysis to multivariate and FDA nonparametric statistics. This package includes the main types of control charts and capability indices. It combines the main features of reputed SQC packages in R such as qcc and qualityTools with the proposal of a new graphical appearance and the implementation of new SQC tools with increasing importance in Industry 4.0 such as multivariate and nonparametric analysis.

In addition to some utilities provided by reference R packages such as qcc, SixSigma, and qualityTools, qcr implements very important statistical techniques of Control and Analysis tasks of the Six Sigma procedure that are not included in other libraries. In the case of multivariate control charts, these tools are the MEWMA and MCUSUM multivariate control charts, on the one hand, and the $r, Q$ and $S$ nonparametric control charts based on data depth, on the other hand. In addition, Phase I and Phase II control charts for functional data (monthly, daily, hourly curves) based on functional data depth, bootstrap procedures, and nonparametric rank charts have also been implemented in the qcr package. These control charts for functional data provide tools to control and improve processes when their CTQ variables are obtained as hourly, monthly, daily, yearly smooth curves.

It is also very important to note that qcr provides functions to perform nonparametric capability analysis. In addition, the new implementation of the process capability plots for the main parametric capability indices allows us to analyze if improvements in process spread or/and process location are needed to obtain a capable process. The comparison between suppliers, machines, etc., is enabled through capability plots.

All these utilities intend to make qcr a useful tool for users of a wide variety of industries, providing a competitive alternative to commercial software.

\section{Acknowledgments}

The work of Salvador Naya, Javier Tarrío-Saavedra, Miguel Flores and Rubén Fernández-Casal has been supported by MINECO grant MTM2017-82724-R, and by the Xunta de Galicia (Grupos de Referencia Competitiva ED431C-2020-14 and Centro de Investigación del Sistema universitario de Galicia ED431G 2019/01), all of them through the ERDF. The research of Miguel Flores has been partially supported by Grant PII-DM-002-2016 of Escuela Politécnica Nacional of Ecuador. In addition, the research of Javier Tarrío-Saavedra has been also founded by the eCOAR project (PC18/03) of CITIC. The authors thank Mateo Larco and Bibiana Santiso for their valuable help with the English edition. 


\section{Bibliography}

F. Barros. IQCC: Improved Quality Control Charts, 2017. URL https://CRAN.R-project.org/ package=IQCC. R package version 0.7. [p196]

A. Bissell. How reliable is your capability index? Applied Statistics, pages 331-340, 1990. [p212]

R. A. Boyles. The Taguchi capability index. Journal of Quality Technology, 23:17-26, 1991. [p196, 212]

D. W. Brown and G. B. Wetherill. Statistical process control. Chapman and Hall, 1990. [p197]

E. L. Cano, J. M. Moguerza, and A. Redchuk. Six Sigma with R, volume 36 of Use R! Springer-Verlag, New York, 2012. [p196]

E. L. Cano, J. M. Moguerza, and M. P. Corcoba. Quality Control with R. Use R! Springer-Verlag, New York, 2015. [p196]

I. Cascos, A. López, and J. Romo. Data depth in multivariate statistics. Boletín de Estadística e Investigación Operativa, 27(3):151-174, 2011. [p200]

C. W. Champ and W. H. Woodall. Exact results for Shewhart control charts with supplementary runs rules. Technometrics, 29(4):393-399, 1987. [p194]

P.-L. Chang and K.-H. Lu. PCI calculations for any shape of distribution with percentile. Quality World Technical Supplement, pages 110-114, 1994. [p211]

Y.-M. Chou, D. Owen, and S. Borrego A. Lower confidence limits on process capability indices. Journal of Quality Technology, 22(3):223-229, 1990. [p212]

A. Cuevas, M. Febrero, and R. Fraiman. Robust estimation and classification for functional data via projection-based depth notions. Computational Statistics, 22(3):481-496, 2007. [p204]

M. Deleryd and K. Vännman. Process capability plots - a quality improvement tool. Quality and Reliability Engineering International, 15(3):213-227, 1999. [p209, 211]

R. Dyckerhoff. Data depths satisfying the projection property. Allgemeines Statistisches Archiv, 88 (2):163-190, 2004. [p200]

M. Flores, S. Naya, R. Fernández-Casal, S. Zaragoza, P. Raña, and J. Tarrío-Saavedra. Constructing a control chart using functional data. Mathematics, 8(1):58, 2020. [p204, 205, 206]

M. Flores, R. Fernández-Casal, S. Naya, and J. Tarrío-Saavedra. qcr: Quality Control Review, 2021. URL https://CRAN.R-project.org/package=qcr. R package version 1.3. [p198, 202]

R. Fraiman and G. Muniz. Trimmed means for functional data. Test, 10(2):419-440, 2001. [p204]

R. Fraiman, R. Y. Liu, and J. Meloche. Multivariate density estimation by probing depth. Lecture Notes-Monograph Series, pages 415-430, 1997. [p200]

A. Gandy and J. T. Kvaloy. Guaranteed conditional performance of control charts via bootstrap methods. Scandinavian Journal of Statistics, 40:647-668, 2013. doi: 10.1002/sjos.12006. [p196]

G. Gruska, K. Mirkhani, and L. Lamberson. Non normal data analysis. St. Clair Shores, MI: Applied Computer Solutions, Inc, 1989. [p212]

N. F. Hubele and K. Vannman. The effect of pooled and un-pooled variance estimators on $c_{p m}$ sub when using subsamples. Journal of quality technology, 36(2):207, 2004. [p211]

S. Knoth. spc: Statistical Process Control - Calculation of ARL and Other Control Chart Performance Measures, 2021. URL https://CRAN.R-project.org/package=spc. R package version 0.6.5. [p196]

R. Liu. On a notion of data depth based on random simplices. The Annals of Statistics, 18(1): 405-414, 1990. [p200]

R. Y. Liu. Control charts for multivariate processes. Journal of the American Statistical Association, 90(432):1380-1387, 1995. [p195, 199, 200] 
R. Y. Liu and J. Tang. Control charts for dependent and independent measurements based on bootstrap methods. Journal of the American Statistical Association, 91(436):1694-1700, 1996. [p197]

P. C. Mahalanobis. On the generalised distance in statistics. Proceedings of the National Institute of Sciences of India, 1936, pages 49-55, 1936. [p200]

D. C. Montgomery. Introduction to statistical quality control. John Wiley \& Sons (New York), 2009. [p194]

P. S. Pande, R. P. Neuman, and R. R. Cavanagh. The six sigma way: How GE, Motorola, and other top companies are honing their performance. McGraw-Hill (New York), 2000. [p194]

W. Pearn and K. Chen. A practical implementation of the process capability index $c_{p k}$. Quality Engineering, 9(4):721-737, 1997. [p211]

A. M. Polansky. Process capability indices, nonparametric. Encyclopedia of Statistics in Quality and Reliability, 2007. [p196]

R Core Team. R: A Language and Environment for Statistical Computing. R Foundation for Statistical Computing, Vienna, Austria, 2021. URL https://www.R-project.org/. [p196]

T. Roth. qualityTools: Statistics in Quality Science., 2016. URL http://www.r-qualitytools.org. R package version 1.55 http://www.r-qualitytools.org. [p196]

E. Santos-Fernandez. Multivariate Statistical Quality Control Using R, volume 14. Springer-Verlag, 2013. ISBN 9781461454533. URL http://www.springer.com/statistics/computational+ statistics/book/978-1-4614-5452-6. [p196, 197]

E. Santos-Fernández and M. Scagliarini. MPCI: An R package for computing multivariate process capability indices. Journal of Statistical Software, 47(7):1-15, 2012. URL http://www . jstatsoft. org/v47/i07/. [p196]

L. Scrucca. qcc: An R package for quality control charting and statistical process control. $R$ News, 4/1:11-17, 2004. URL https://cran.r-project.org/doc/Rnews/. [p196, 197]

L.-I. Tong and J.-P. Chen. Lower confidence limits of process capability indices for non-normal process distributions. International Journal of Quality $\& \mathcal{G}$ Reliability Management, 15(8/9):907-919, 1998. [p211, 212]

J. W. Tukey. Mathematics and the picturing of data. In Proceedings of the international congress of mathematicians, volume 2, pages 523-531, 1975. [p200]

K. Vännman. A unified approach to capability indices. Statistica Sinica, pages 805-820, 1995. [p208]

K. Vännman. A graphical method to control process capability. In Frontiers in Statistical Quality Control 6, pages 290-311. Springer-Verlag, 2001. [p209, 211]

W. Zhu and C. Park. edcc: An R package for the economic design of the control chart. Journal of Statistical Software, 52(9):1-24, 2013. URL http://www.jstatsoft.org/v52/i09/. [p196]

Y. Zuo and R. Serfling. General notions of statistical depth function. Annals of statistics, pages 461-482, 2000. [p200]

\author{
Miguel Flores \\ 170517 Quito \\ Ecuador \\ 0000-0002-7742-1247 \\ miguel.flores@epn.edu.ec \\ Rubén Fernández-Casal \\ MODES, CITIC, Universidade da Coruña \\ Facultade de Informática, Campus de Elviña, A Coruña \\ Spain \\ 0000-0002-5785-3739 \\ ruben.fcasaloudc.es
}

MODES, SIGTI, ADIAAC, Departamento de Matemáticas, Escuela Politécnica Nacional 
Salvador Naya

MODES, CITIC, ITMATI, Universidade da Coruña Escola Politécnica Superior, Mendizábal s/n, Ferrol Spain

0000-0003-4931-9859

salva@udc.es

Javier Tarrío-Saavedra

MODES, CITIC, Universidade da Coruña

Escola Politécnica Superior, Mendizábal s/n, Ferrol

Spain

0000-0003-4931-9859

javier.tarrio@udc.es 